\title{
A Harmonic Resonance Suppression Strategy for a High-Speed Railway Traction Power Supply System with a SHE-PWM Four-Quadrant Converter Based on Active-Set Secondary Optimization
}

\author{
Runze Zhang ${ }^{1}$, Fei Lin ${ }^{1}$, , Zhongping Yang ${ }^{1}$, Hu Cao ${ }^{2}$ and Yuping Liu ${ }^{2}$ \\ 1 School of Electrical Engineering, Beijing Jiaotong University, Beijing 100089, China; \\ 16126069@bjtu.edu.cn (R.Z.); zhpyang@bjtu.edu.cn (Z.Y.) \\ 2 CCRC Qingdao Sifang Rolling Stock Research Institute Co. Ltd., Qingdao 266000, China; \\ cao_hu123@126.com (H.C.); 13792844397@163.com (Y.L.) \\ * Correspondence: flin@bjtu.edu.cn; Tel.: +86-136-4139-8863
}

Received: 19 September 2017; Accepted: 2 October 2017; Published: 11 October 2017

\begin{abstract}
Pulse width modulation (PWM) technology is widely used in traction converters for high-speed railways. The harmonic distribution caused by PWM is quite extensive, and increases the possibility of grid-train coupling resonance in the traction power supply system (TPSS). This paper first analyzes the mechanism of resonance, when the characteristic harmonic frequency of a four-quadrant converter $(4 \mathrm{QC})$ current that injects into the traction grid matches the resonant frequency of the traction grid, which may result in resonance in the system. To suppress resonance, this paper adopts specific harmonic elimination-pulse width modulation (SHE-PWM) technology combined with a transient direct current control strategy to eliminate the harmonics in the resonant frequency, which may suppress the grid-train coupling resonance. Due to the fact that the SHE-PWM process with multiple switching angles contains complex transcendental equations, the initial value is difficult to provide, and is difficult to solve using ordinary iterative algorithms. In this paper, an active-set secondary optimization method is used to solve the equation. The algorithm has the benefits of low dependence on initial values, fast convergence and high solution accuracy. Finally, the feasibility of the resonant suppression algorithm is verified by means of Matlab simulation.
\end{abstract}

Keywords: high-speed railway; pulse width modulation (PWM); harmonic resonance suppression; specific harmonic elimination-pulse width modulation (SHE-PWM); active set

\section{Introduction}

Sinusoidal pulse width modulation (PWM) technology has been used in electrified railway systems for more than 20 years. This technology produces high-frequency harmonics, which are injected into the traction power supply grid through the pantograph [1-6]. The traction power supply system (TPSS) can be equivalent to a distributed parameter system. When the transformer leakage inductance matches the traction grid impedance, and the frequency of harmonic current that is injected into the traction grid is equal to the resonance frequency of the traction grid, a phenomenon of harmonic amplification will occur, which will result in the distortion of the grid voltage.

At present, there are two main methods by which to suppress grid-train coupling resonance: changing the traction grid impedance, and improving the harmonic current that is injected into the TPSS. Changing the impedance characteristics of the traction grid is described in $[7,8]$. The main method is to install the resistor on the line. This has been applied on the actual line and achieved modest effects. However, this method will increase the line cost and alter the original electrical characteristics of the line. The representation method of improving the current injected into the traction 
grid is adding an active filter [9-12]; this method can be further divided into two types: ground style and train active filter. However, the existing active filter functions are mostly focused on optimizing the quality of electrified railway power, not the resonance problem of the traction grid.

In terms of improving the current which injected into traction grid method, [13] altered the converter switching frequency to avoid the resonant frequency of the power supply line. However, in actual working conditions, the range of the converter switching frequency cannot change widely, thus this method cannot be used in practice. Cui et al. [3,14] proposed the use of specific harmonic elimination pulse width modulation (SHE-PWM) technology to improve the harmonic current of the traction grid. SHE-PWM technology was first proposed by Patel in 1973 [15]. According to the different positions of the pulse wave switching angle, the basic component is controlled and the specific subharmonic is eliminated. Due to its precise control of the harmonics, this method is rapidly applied in the fields of AC speed control and multi-level converters [16-18]. The difficulty of the SHE-WPM method is that the challenge of solving the switching angle equation increases greatly with an increasing number of switching angles. In $[3,14]$, the process of solving the transcendental equation of SHE-PWM is not described in detail, but these two papers provide valuable ideas for the writing of the present one.

The SHE-PWM equation consists of a series of transcendental equations composed of trigonometric functions. The solving process is simple when the number of switching angles is small, and the number of harmonic elimination is continuous. When the number of switching angles is increased and the number of elimination harmonics is set to be complicated, the difficulty will increase significantly. Dahidah et al. [19] introduced the existing methods of solving SHE-PWM equations, such as Newton iteration, particle swarm optimization, and so on. In the above solving methods, there are some problems such as serious dependence on the initial values setting and complex solution process, and thus they are not suitable for the condition of complex switching angle and harmonic elimination. In this paper, based on the active set algorithm, the SHE-PWM equation is solved by using the quadratic optimization iterative method, which can eliminate the dependence of the given initial value, accelerate the iterative convergence speed, and obtain a high-precision solution.

In using the SHE-PWM method, this paper focuses on a train operating at high speed and in a steady-state process. The current harmonic of the main transformer primary side on the train is unstable and the modulation index varies greatly during the starting phases of the train. Saponara et al. [20] described the EMI (Electromagnetic Interference) problem of electric vehicles in the start-up process, which has significance for the study of the train starting process. In this paper, the train starting process will not be studied; it will be followed up on in future research.

The second section of this paper will first introduce the transient direct current control strategy of a four-quadrant converter ( $4 \mathrm{QC})$, derive a harmonic equation of $4 \mathrm{QC}$, explain the mechanism of the grid-train coupling resonance, and propose a harmonic suppression strategy based on the specific harmonic elimination technique. The third section first gives a brief overview of the basic principles of SHE-PWM, and then introduces in detail the specific harmonic elimination strategy based on the multiplex 4QC. Next, it presents the solving algorithm of a transcendental equation, and further explains the block diagram of SHE-PWM combined with transient direct current control. The fourth section verifies the resonance suppression strategy by means of simulation results and control strategy. Finally, we provide a summary of the whole paper.

\section{Grid-Train Coupling Resonance Mechanism and Suppression Method}

A diagram of the traction drive system is shown in Figure 1. In practice, multiple traction converters run in parallel and only one is shown in Figure 1. As in the middle of the DC (Direct Current) link, a large capacitor is generally chosen as a support capacitor; the inverter and motor load on the front four quadrant converter harmonic effects can be ignored. Therefore, in the study of grid-train coupling systems, the equal power 4QC model is adopted to replace the train model for 
grid-train coupling analysis. Different $4 \mathrm{QC}$ modulation algorithms have different harmonic effects on the grid-train coupling system.

In this paper, the transient direct current control strategy is used as an example to introduce the $4 \mathrm{QC}$ harmonic distribution.

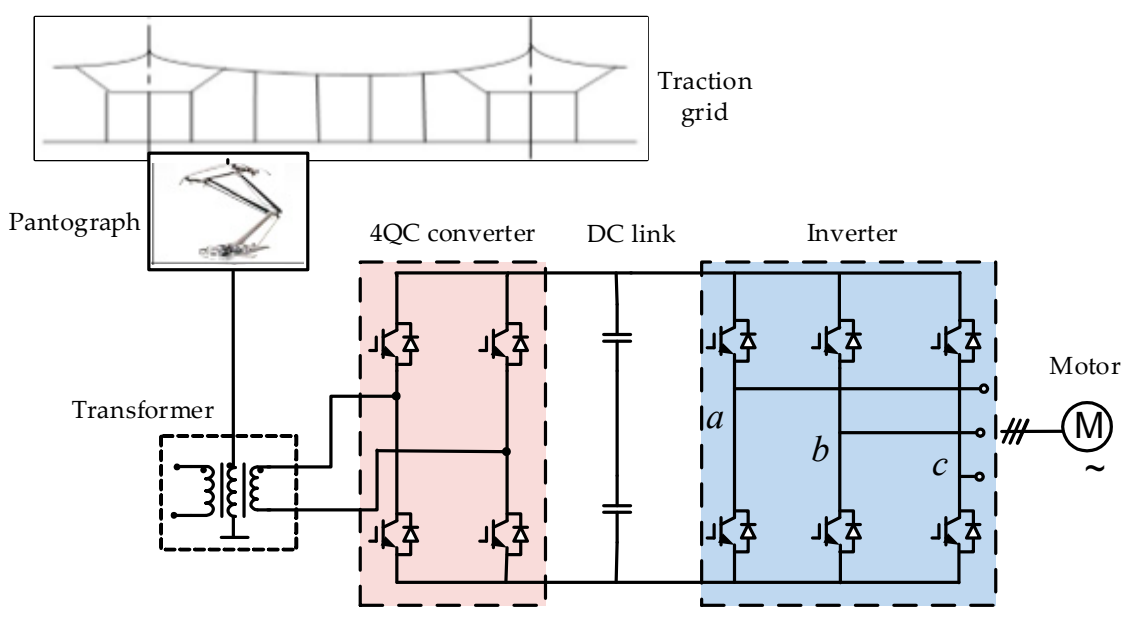

Figure 1. Diagram of traction drive system.

\subsection{Introduction of Transient Direct Current Control Strategy for Four-Quadrant Converter}

The high-speed railway train single-phase two-level four-quadrant converter topology and control block diagram are shown in Figure 2. The $u_{N}$ and $i_{N}$ indicate the voltage and current on the grid side, respectively; $U_{d}$ is the DC side voltage; $L$ is the filter inductance on the input side, which is composed of the leakage inductance in the transformer; $C$ is the DC side support capacitor; and $R_{L}$ is the equivalent DC load.

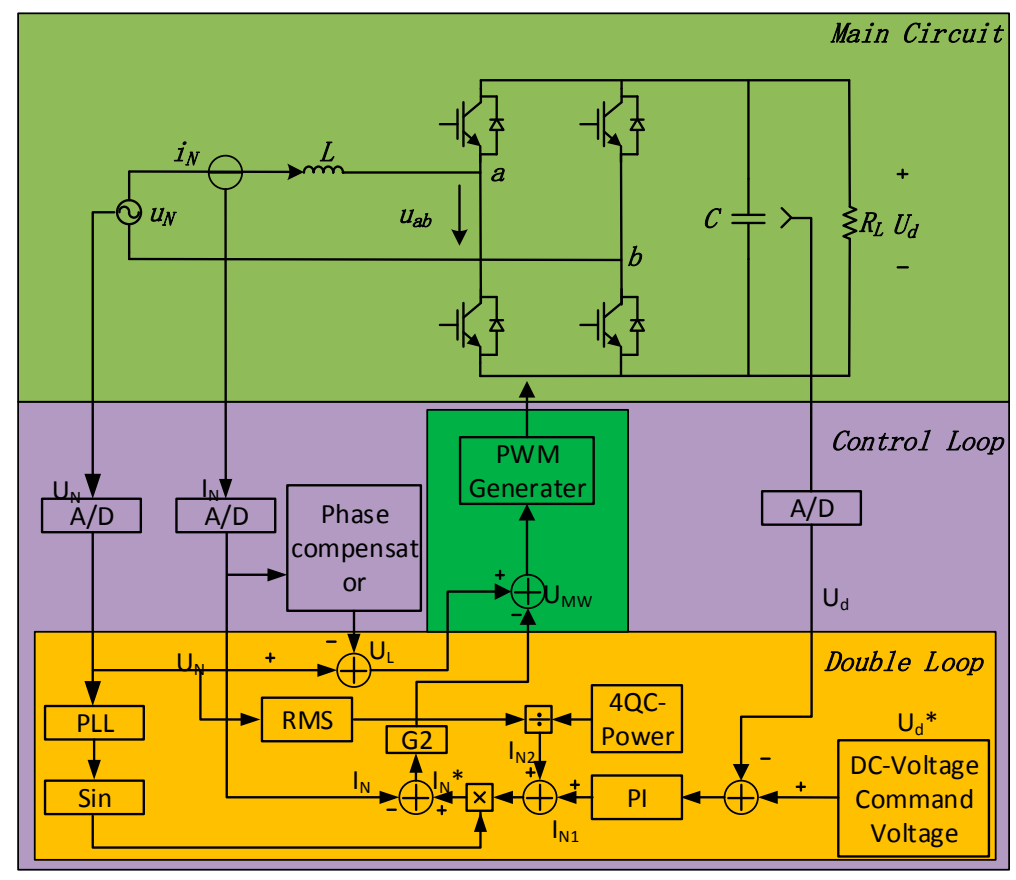

Figure 2. Diagram of four-quadrant converter (4QC). 
The 4QC usually adopts a double closed-loop control mode, which combines a voltage outer loop and current inner loop. The transient direct current control block diagram is shown in the lower half of Figure 2.

The mathematical model is as follows:

$$
\left\{\begin{array}{l}
I_{N 1}=k_{p}\left(U_{d}{ }^{*}-U_{d}\right)+\frac{1}{T_{i}} \int\left(U_{d}{ }^{*}-U_{d}\right) d t \\
I_{N 2}=\frac{I_{d} U_{d}}{U_{N}} \\
I_{N}{ }^{*}=I_{N 1}+I_{N 2} \\
u_{M W}(t)=u_{N}(t)-\omega_{m} L_{N} I_{N}{ }^{*} \cos \omega_{m} t-G_{2}\left[I_{N}{ }^{*} \sin \omega_{m} t-i_{N}(t)\right]
\end{array}\right.
$$

The voltage outer loop adopts PI (Proportional Integral) controller to adjust the actual DC voltage $U_{d}$ so that it can track its reference value $U_{d}{ }^{*}$. Its output is the fluctuation component of the AC current reference amplitude $I_{N 1}$, and the steady-state component of the AC current reference value is calculated by the disturbance feed forward $I_{N 2}$, which can be used to reduce the working pressure of the PI controller and prevent the PI controller from reaching saturation. The principle of calculating $I_{N 2}$ is that the input power of the grid side and the load consumption power are balanced in the steady state. The sum of the $I_{N 1}$ and $I_{N 2}$ is the reference amplitude of the AC current $I_{N}{ }^{*}$. The reference value of the inner loop AC current is obtained by multiplying the current reference amplitude $I_{N}{ }^{*}$ by the grid voltage phase. This value contains information that enables the DC voltage to follow the given value and the AC current to track the voltage phase of the grid side. The current loop uses a proportional controller to regulate the actual AC current so that it can track its reference value. A steady-state component perturbation $u_{N}-u_{L}$ feed forward is introduced, where $u_{N}$ is the grid voltage, which is measured directly. The inductance voltage $u_{L}$ is obtained by current reference estimation. In the steady state, the actual current will track the reference current. The output of the current loop $u_{M W}$ is the reference value of the AC side voltage of the single-phase $4 \mathrm{QC}$, which is the reference signal of the PWM modulation section.

\subsection{Harmonic Distribution of Four Quadrant Converter}

The two-level four-quadrant converter modulation principle is shown in Figure 3. The sinusoidal modulated wave is compared with the triangular carrier to obtain the control pulse signal of the bridge arm.

The respective expressions of the phase A and B modulation waves are as follows:

$$
\left\{\begin{array}{l}
f_{m a}(t)=M \cos \left(\omega_{m} t+\beta\right) \\
f_{m b}(t)=-M \cos \left(\omega_{m} t+\beta\right)
\end{array}\right.
$$

In the equation, $M$ is the modulation index, which is obtained by comparing the fundamental amplitude of the modulation wave in Figure 2 with the DC side voltage; $\omega_{m}$ is the modulation wave frequency, and $\beta$ is the initial phase angle of the modulation waveform.

The carriers of phase A and B are same, and the expression is as follows:

$$
f_{c}(t)= \begin{cases}-\left(\frac{2 \omega_{c}}{\pi} t+\frac{2 \alpha}{\pi}\right)+1+4 p & t \in\left[\frac{2 p \pi}{\omega_{c}}, \frac{(2 p+1) \pi}{\omega_{c}}\right] \\ \left(\frac{2 \omega_{c}}{\pi} t+\frac{2 \alpha}{\pi}\right)-3-4 p & t \in\left[\frac{(2 p+1) \pi}{\omega_{c}}, \frac{(2 p+2) \pi}{\omega_{c}}\right]\end{cases}
$$

where $\omega_{c}$ is the carrier frequency, and $\alpha$ is the carrier phase angle, $p=0,1,2 \ldots$ 

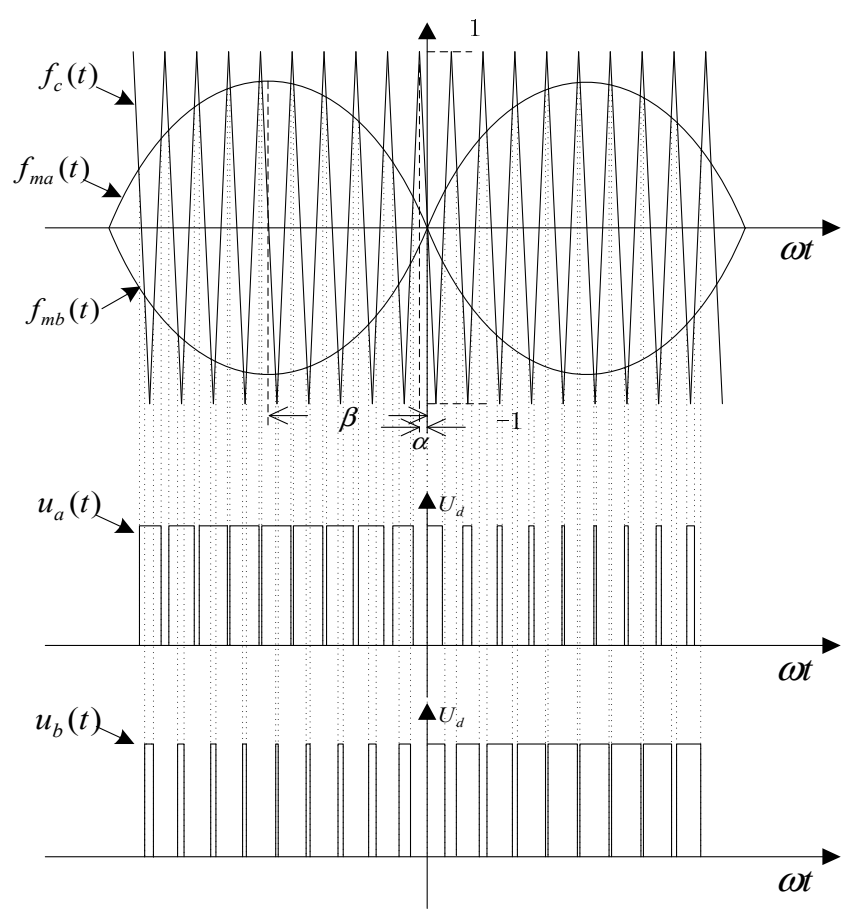

Figure 3. Two-level 4QC modulation principle.

In a modulation wave period, PWM pulse $u_{a}(t)$ Fourier analysis can be performed, after which the correlation coefficients in the bilateral Fourier equation are obtained. According to the bilateral Fourier equation, the expression of the voltage $u_{a}(t)$ of the phase A bridge arm can be obtained. As the phase modulation wave of phase $B$ is reversed with phase $A$, the phase difference is $180^{\circ}$. Therefore, the Fourier equation of $u_{b}(t)$ can be obtained by writing $\beta$ into $\beta+\pi$. Then the expression of the pulse voltage $u_{a b}$ can be obtained as follows:

$$
\begin{aligned}
& u_{a b}(t)=u_{a}(t)-u_{b}(t)=M U_{d} \cos \left(\omega_{m} t+\beta\right)+ \\
& \sum_{m=2,4 \ldots n= \pm 1, \pm 3 \ldots}^{\infty} \frac{4 U_{d}}{m \pi} \sin \frac{n \pi}{2} \cos \frac{m \pi}{2} J_{n}\left(\frac{m \pi M}{2}\right) \cos \left(m \omega_{c} t+n \omega_{m} t+m \alpha+n \beta\right)
\end{aligned}
$$

where $J_{n}$ is the Bessel function.

From the diagram of the $4 \mathrm{QC}$ shown in Figure 1, the voltage vector relationship is as follows:

$$
\overrightarrow{u_{N}}=\overrightarrow{u_{L}}+\overrightarrow{u_{a b}}
$$

The inductance voltage $u_{L}$ can be expressed as:

$$
u_{L}=L \frac{d i_{N}}{d t}
$$

The expression of the input current $i_{N}$ of the $4 \mathrm{QC}$ can be calculated from the above equation.

$$
\begin{aligned}
& i_{N}(t)=\frac{\sqrt{M^{2} U_{d}^{2}-2 U_{N}{ }^{2}}}{\omega_{m} L_{N}} \cos \left(\omega_{m} t+\beta\right)+ \\
& \sum_{m=2,4 \ldots}^{\infty} \sum_{n= \pm 1, \pm 3 \ldots}^{\infty} \frac{4 U_{d}}{m \pi L_{N}\left(m \omega_{c}+n \omega_{m}\right)} J_{n}\left(\frac{m \pi M}{2}\right) \cos \frac{m \pi}{2} \sin \frac{n \pi}{2} \sin \left(m \alpha+n \beta+m \omega_{c} t+n \omega_{m} t\right)
\end{aligned}
$$

From the above equation, it can be seen that the input current contains a large amount of harmonic components aside from the fundamental component, which has the same frequency as the traction grid voltage. Ideally, the harmonic components are as summarized below: 
(1) The high-frequency harmonic component in the input current is concentrated in an even number of times near the switching frequency;

(2) $m \omega_{c}+n \omega_{m}$ is odd, which signifies that the harmonics do not contain even harmonics;

(3) The larger the $m$ value in the equation, the smaller the harmonic content and amplitude will be, which means that the content and amplitude of the harmonics decay rapidly with the increase in harmonic order;

(4) The harmonic content is affected by the modulation index, magnitude of the DC voltage, root mean square voltage of the grid side, grid voltage frequency and PWM modulation carrier frequency, and the amplitude of each harmonic is also related to the inductance of the AC side of the $4 \mathrm{QC}$.

In actual working conditions, a multi-carrier phase shift strategy is often adopted to control the multiple 4QC on the transformer secondary side, and each 4QC still uses the transient direct current control strategy. Therefore, the harmonic characteristics of the transformer primary current are similar to those described above. Only due to the carrier phase shift strategy will the equivalent switching frequency be increased, after which the corresponding harmonic band will shift to the right. The specific equation is not repeated in this paper.

\subsection{Grid-Train Coupling Resonance Mechanism}

A diagram of a double track AT TPSS is shown in Figure 4. There is a coupling relationship that has mutual impedance and stray capacitance between the actual line conductors. As shown in Figure 5, it is an impedance characteristic analysis diagram of a typical TPSS. It can be seen that this is a multi-impedance peak system. In order to simplify the analysis, Figure 4 can be equivalent to the schematic diagram of the simplified traction grid shown in Figure 6.

The train model is equivalent to a $4 \mathrm{QC}$ to reflect the harmonic characteristics of the entire train to the traction grid. $Z_{L}$ is the equivalent impedance of the traction grid on the left of the train; $Z_{R}$ is the equivalent impedance of the traction grid on the right side of the train; $Z_{i}$ is the equivalent impedance of the entire traction grid seen from the train; $L_{N}$ and $R_{N}$ are the transformer impedance equivalents to the primary side of the transformer; $U_{N}$ is the traction grid voltage; $i_{N}$ is the current that is injected into the traction grid by the $4 \mathrm{QC}$; and $u_{a b}$ is the pulse voltage of the $4 \mathrm{QC}$.

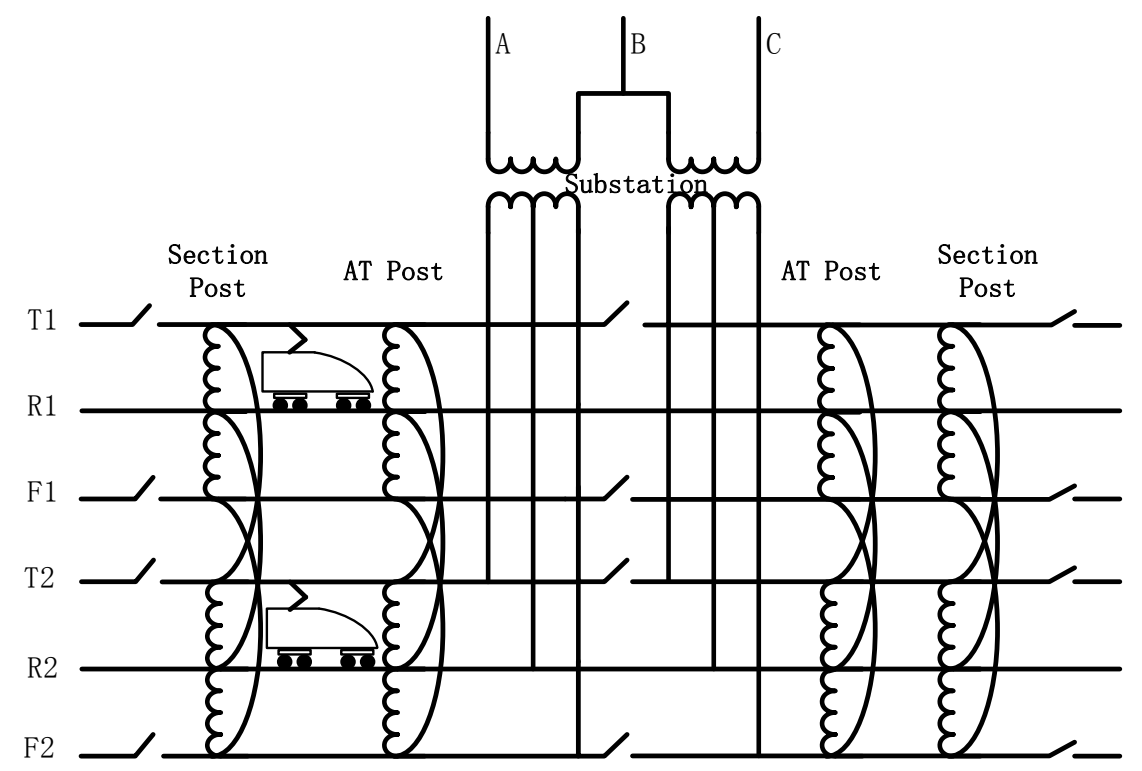

Figure 4. AT TPSS diagram. 
When the train runs in different positions on the traction grid, the traction grid equivalent impedance $Z_{L}$ and $Z_{R}$ values also change. For the harmonic of the $4 Q C$ injected into the traction grid, when the impedance of one side of the train is capacitive and the other side is inductive, the grid-train coupling model shown in Figure 6 may be equivalent to the parallel impedance resonant analysis circuit shown in Figure 7.

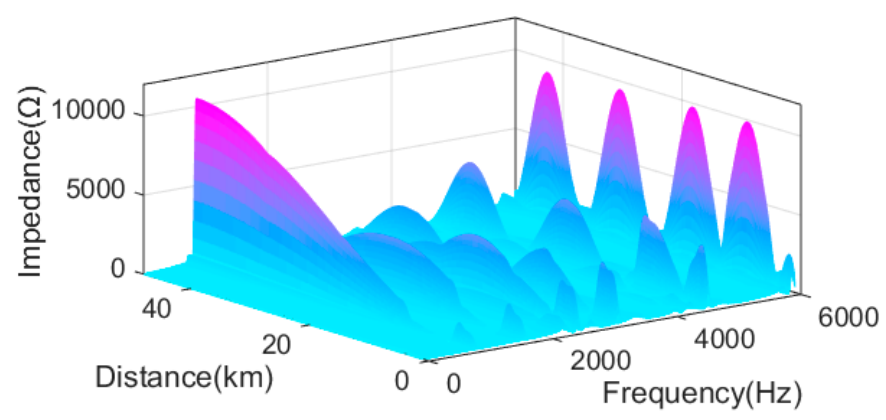

Figure 5. Impedance analysis of traction power supply system.

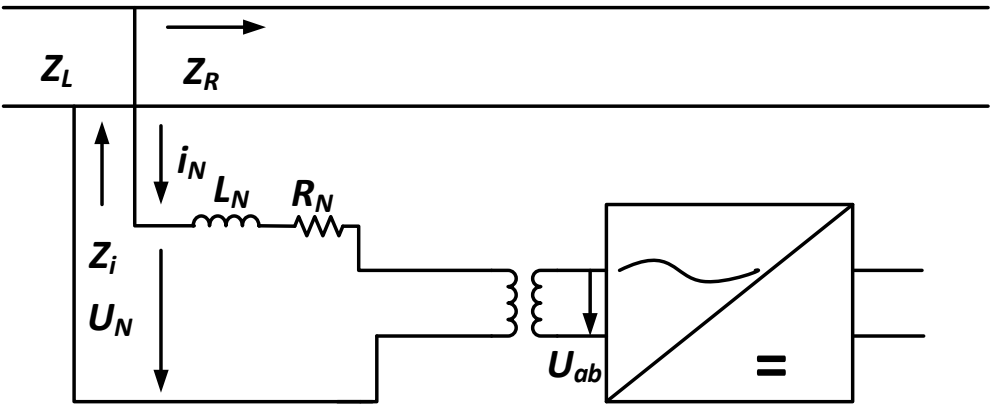

Figure 6. Grid-train coupling diagram.

In Figure $7, R_{1}$ and $C_{1}$ are the capacitive impedance values of the traction grid on one side of the train, while $R_{2}$ and $L_{2}$ are the inductive impedance values of the traction grid on the other side. The left and right side impedance parameters of the train, namely $R_{1} / C_{1}$ and $R_{2} / L_{2}$, have a specific resonance frequency that is set as $\omega_{r p}$, at which time the equivalent impedance value of traction grid $Z_{i}$ is taken as the maximum value, and shows pure resistivity.

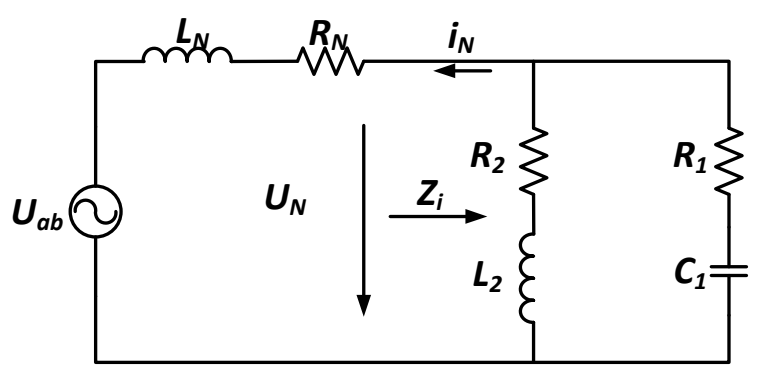

Figure 7. Equivalent parallel resonant circuit.

When the resonance occurs, the harmonic voltage in the grid voltage is as follows:

$$
U_{N(\omega r p)}=i_{N(\omega r p)} Z_{i(\omega r p)}
$$

When the characteristic harmonic frequency of the current $i_{N}$ that is injected into the traction grid is equal to the resonant frequency of the traction grid, such as the resonant frequency shown in 
Figure 5, the harmonic content of the grid voltage $U_{N}$ will rise significantly. The superposition of the harmonic voltage and fundamental voltage can cause a distortion of the traction grid voltage and affect the normal operation of the system.

It can be seen from Equation (8) that there are two main factors affecting the resonance. One is the harmonic current with the same frequency as the resonance, and the other is the traction grid impedance peak on the resonant frequency. This paper uses SHE-PWM technology to control the 4QC so as to eliminate the harmonic current with the resonance frequency injected into the traction grid. Figure 8 shows a flow diagram of the resonance suppression.

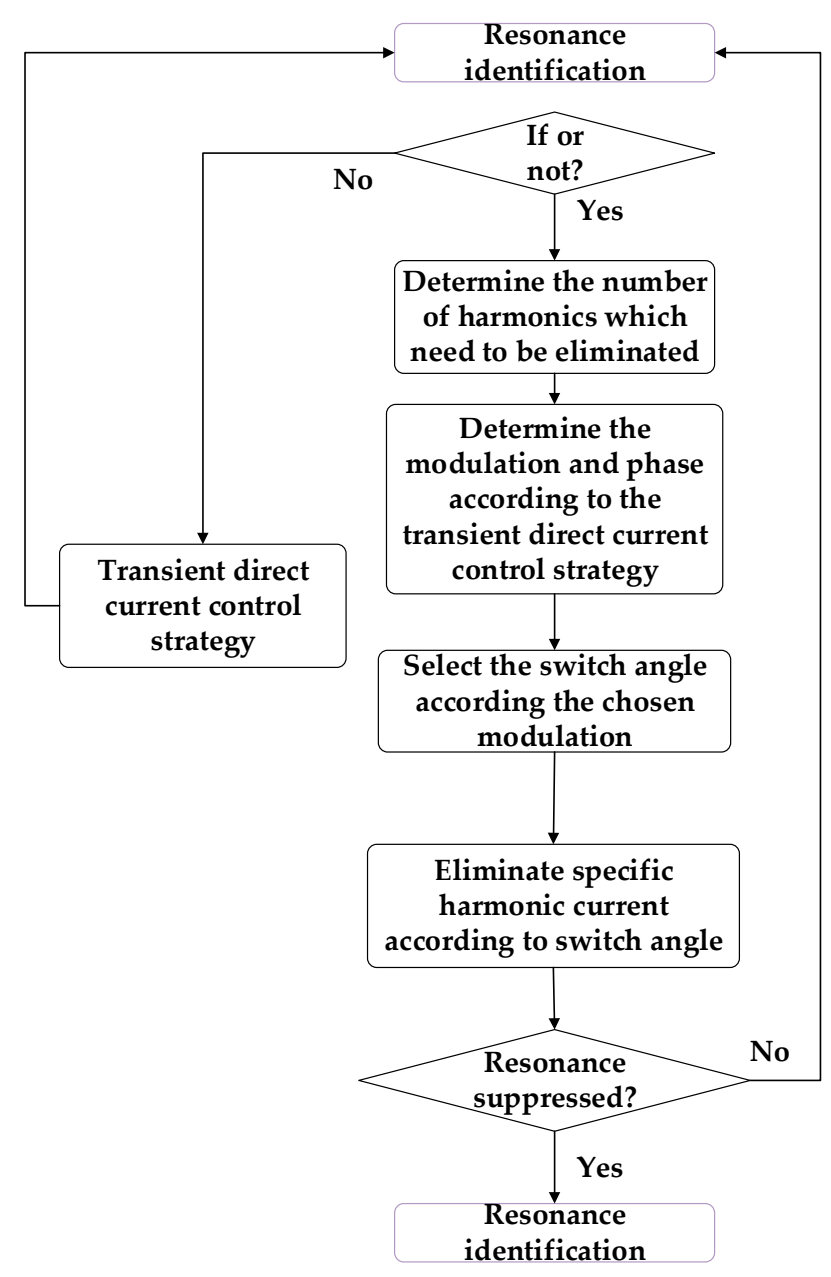

Figure 8. Flow chart of harmonic suppression.

\section{Resonance Suppression Strategy and Equation Solution Based on Specific Harmonic Elimination-Pulse Width Modulation}

In the $4 \mathrm{QC}$, the current harmonic distribution at the primary side of the transformer can be controlled by controlling pulse voltage between the two-phase bridge arms using the SHE-PWM method.

\subsection{Fundamental of Specific Harmonic Elimination-Pulse Width Modulation}

First, we consider a simple $4 \mathrm{QC}$ topology whose AC side pulse voltage $u_{a b}$ can be represented by a three-level pulse, as shown in Figure 9. 


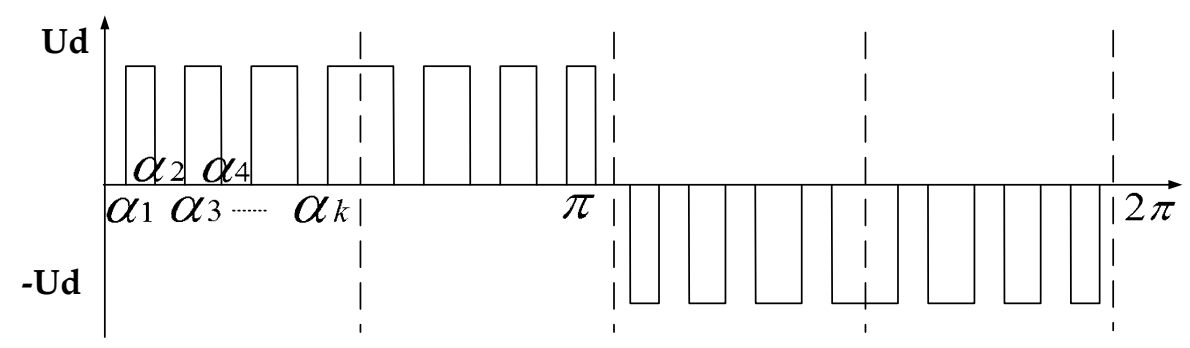

Figure 9. Three-level pulse waveform.

Among them, $\alpha_{1}, \alpha_{2} \ldots \alpha_{k}$ are the switching angles of the $1 / 4$ switching pulse period. Assuming that the pulse waveform is $1 / 4$ periodic symmetrical, the Fourier analysis of the pulse voltage in Figure 9 is shown as follows:

$$
\begin{gathered}
U_{a b}=\sum_{n=1}^{\infty}\left[a_{n} \cos (n \omega t)+b_{n} \sin (n \omega t)\right] \\
\left\{\begin{array}{l}
a_{n}=\frac{2}{n \pi} U_{d} \sum_{i=1}^{k}(-1)^{k+1} \sin \left(n \alpha_{k}\right) \\
b_{n}=\frac{2}{n \pi} U_{d} \sum_{i=1}^{k}(-1)^{k+1} \cos \left(n \alpha_{k}\right)
\end{array}\right.
\end{gathered}
$$

In the equation, $n$ is the harmonic order, and $u_{d}$ is the DC side voltage.

Due to the fact that the pulse voltage is $1 / 4$ periodic symmetrical, the DC component and even number of times harmonic component are zero, $a_{n}=0$. It is only necessary to consider the odd number of times harmonic component values.

The value of the fundamental component of the pulse voltage $u_{a b}$ divided by the DC side voltage is defined as the modulation index $\mathrm{M}$, which is as follows:

$$
M=\frac{b_{1}}{U_{d}}
$$

Considering the $4 \mathrm{QC}$ input and output requirements, in addition to making the fundamental component satisfy the modulation requirements, it also can eliminate up to $k-1$ odd number of times harmonic orders, the expression for which is as follows:

$$
\left\{\begin{array}{c}
\sum_{i=1}^{k}(-1)^{i+1} \cos \left(\alpha_{i}\right)=\frac{\pi}{4} M \\
\sum_{i=1}^{k}(-1)^{i+1} \cos \left(n \alpha_{i}\right)=0
\end{array}\right.
$$

The switching angles requirements are: $0<\alpha_{1}<\alpha_{2}<\cdots<\alpha_{2}<\frac{\pi}{2}$.

In general SHE-PWM problems, the odd number of times harmonic, such as $n=3,5,7, \ldots, 2(k-1)$ -1 , is selected as the selected harmonic elimination times. Therefore, according to the constraint conditions and number of selected harmonic eliminations, it is necessary to solve the angle that satisfies Equation (12), namely the mathematical description of the SHE-PWM method.

\subsection{Specific Harmonic Elimination Strategy of Multi-Four-Quadrant Converter}

In the existing high-speed railway system, the traction transformer of the train generally contains a small number of secondary windings, and the $4 \mathrm{QC}$ is carried by the secondary windings as a whole, using multiple strategies to optimize the control. Multiplexing technology has the advantages of increasing the capacity of the device, increasing the equivalent switching frequency of the converter, 
and reducing the harmonic current of the converter input current. This paper takes a quadruple $4 \mathrm{QC}$ as an example to discuss the control strategy, in which the switching frequency is set to $350 \mathrm{~Hz}$.

For a single $4 \mathrm{QC}$, when the switching frequency is $350 \mathrm{~Hz}$, there are seven controllable switching angles in the $1 / 4$ switching cycle, and in a quadruple 4QC there are 28 controllable switching angles in the $1 / 4$ cycle. Assuming that the quadruple $4 \mathrm{QC}$ has the same modulation index, according to Equation (12), for the fundamental of the pulse voltage, the equation is as follows:

$$
\begin{aligned}
& {\left[\sum_{i=1}^{7}(-1)^{i+1} \cos \alpha_{i}-\frac{\pi}{4} \mathrm{M}\right]^{2}+\left[\sum_{i=8}^{14}(-1)^{i} \cos \alpha_{i}-\frac{\pi}{4} \mathrm{M}\right]^{2}+} \\
& {\left[\sum_{i=15}^{21}(-1)^{i+1} \cos \alpha_{i}-\frac{\pi}{4} \mathrm{M}\right]^{2}+\left[\sum_{i=22}^{28}(-1)^{i} \cos \alpha_{i}-\frac{\pi}{4} \mathrm{M}\right]^{2}=0}
\end{aligned}
$$

where $M$ is the modulation index shown in Equation (11), and its value ranges from 0.5 to 1.0.

For the harmonic control, it can be achieved by controlling the synthesis PWM of the quadruple $4 \mathrm{QC}$ :

$$
\left[\sum_{i=1}^{7}(-1)^{i+1} \cos n \alpha_{i}+\sum_{i=8}^{14}(-1)^{i} \cos n \alpha_{i}+\sum_{i=15}^{21}(-1)^{i+1} \cos n \alpha_{i}+\sum_{i=22}^{28}(-1)^{i} \cos n \alpha_{i}\right]^{2}=0
$$

At this point, each 4QC must satisfy the following constraints of the switching angles:

$$
\left\{\begin{array}{l}
0<\alpha_{1}<\alpha_{2}<\cdots<\alpha_{7}<\frac{\pi}{2} \\
0<\alpha_{8}<\alpha_{9}<\cdots<\alpha_{14}<\frac{\pi}{2} \\
0<\alpha_{15}<\alpha_{16}<\cdots<\alpha_{21}<\frac{\pi}{2} \\
0<\alpha_{22}<\alpha_{23}<\cdots<\alpha_{28}<\frac{\pi}{2}
\end{array}\right.
$$

According to the above analysis, in the 28 controllable switching angles of the quadruple $4 \mathrm{QC}$, four angles are required to control the fundamental component of the quadruple $4 \mathrm{QC}$, thus there are at most 24 harmonic orders that can be controlled. In solving the multi-level SHE-PWM equation, it is necessary to satisfy the elimination of the selected harmonics and control all four 4QC modulation requirements at the same time. As a result, no switching angle solution may occur at some modulation index working points. In [21], it was proposed that the freedom degree of the solution can be increased by reducing the harmonic elimination numbers. In this paper, the range and continuity of the solution can be improved by reducing two elimination harmonic orders and controlling the 22 odd harmonic orders.

As the existing grid-train coupling resonance occurs in three bands, i.e., high-, medium- and low-frequency. Therefore, when choosing the harmonic elimination frequency, it is not advisable to choose a strategy to select the number of harmonics from the third harmonic; instead it should cover the different bands of harmonics. Clearly, the low switching frequency $(250-550 \mathrm{~Hz})$ in high power cannot provide enough switching angles to eliminate all potential resonant frequencies. In [14], it was proposed to use the segmented harmonic elimination method to achieve full band coverage. In this paper, a similar method is adopted to select harmonic elimination orders.

In order to achieve higher harmonic order coverage, the harmonic elimination orders are divided into four groups, and each group is divided into two parts: BF 1 (Band Frequency 1) and BF 2.x (Band Frequency 2.x), as shown in Figure 10. BF 1 eliminates the low-frequency third to 35th harmonics, and BF 2 is divided into four subintervals. The first subinterval eliminates the 37th to 45th harmonics, and the fourth subinterval is the 67th to 75 th harmonics. 


\begin{tabular}{cccccc|} 
Band & Band & Band & Band & Band \\
1 & 2.1 & 2.2 & 2.3 & 2.4 \\
\hline $3-\ldots---35$ & $37 \cdots \cdots .45$ & $47 \cdots \cdots 5$ & $57 \cdots \cdots .65$ & $67 \cdots \cdots \cdot 75$
\end{tabular}

Figure 10. Schematic diagram of harmonic elimination.

The harmonic elimination method is as follows:

(1) Select four switching angles by which to control the four fundamental components of the quadruple 4QC;

(2) Abandon two harmonic order controls, in order to obtain a higher tolerance so as to solve the transcendental equation;

(3) Among the remaining 22 switching angles, select 17 angles to eliminate the third to 35th harmonics; the remaining five angles are assigned to BF 2.x to eliminate high-frequency harmonics.

According to the aforementioned harmonic selection allocation scheme, the problem of solving the aforementioned transcendental equation can be described as a nonlinear optimization problem combining Equations (13) and (14). This is shown in Equation (16):

$$
\begin{aligned}
& P_{\min }=\left[\sum_{i=1}^{7}(-1)^{i+1} \cos \alpha_{i}-\frac{\pi}{4} M\right]^{2}+\left[\sum_{i=8}^{14}(-1)^{i} \cos \alpha_{i}-\frac{\pi}{4} M\right]^{2}+\left[\sum_{i=15}^{21}(-1)^{i+1} \cos \alpha_{i}-\frac{\pi}{4} M\right]^{2}+ \\
& {\left[\sum_{i=22}^{28}(-1)^{i} \cos \alpha_{i}-\frac{\pi}{4} M\right]+\sum_{n}\left[\sum_{i=1}^{7}(-1)^{i+1} \cos n \alpha_{i}+\sum_{i=8}^{14}(-1)^{i} \cos n \alpha_{i}+\sum_{i=15}^{21}(-1)^{i+1} \cos n \alpha_{i}+\sum_{i=22}^{28}(-1)^{i} \cos n \alpha_{i}\right]^{2}}
\end{aligned}
$$

The modulation index $0.5 \leq M \leq 1.0$, and the constraint condition of the switching angles is the same as that shown in Equation (15). In Equation (16), the order of elimination harmonic $n$ is selected as follows:

$$
n_{1}=\left\{\begin{array}{l}
3,5,7,9, \ldots, 33,35 \\
37,39,41,43,45 \\
3,5,7,9, \ldots, 33,35 \\
57,59,61,63,65
\end{array}, n_{3}=\left\{\begin{array}{l}
3,5,7,9, \ldots, 33,35 \\
47,49,51,53,55 \\
3,5,7,9, \ldots, 33,35 \\
67,69,71,73,75
\end{array}\right.\right.
$$

\subsection{Specific Harmonic Elimination-Pulse Width Modulation Transcendental Equation Solving Process}

There are many methods for solving nonlinear transcendental equations, most of which involve numerical iteration. In [19], a solving method of SHE-PWM equation composed of a cosine function was reviewed. The Newton iteration method, Walsh function method, particle swarm optimization, and genetic algorithm are also applied to the numerical solution. However, the above method is mainly used for equations in which the switching angles are relatively small, and there is some difference between the elimination strategies introduced in Section 3.2. Therefore, this paper presents a numerical iterative method based on the active set algorithms to obtain the solution of the cosine transcendental equation with multi-switching angles and intermittent harmonic elimination.

The general constrained optimization problem is expressed in the following equation:

$$
\left\{\begin{array}{l}
\min \quad P(\alpha) \\
\text { s.t } \quad l \leq \alpha \leq u
\end{array}\right.
$$

In the above equation, $P(\alpha): R^{n} \rightarrow R$ is a real value function, $\alpha, l, u \in R^{n}$, and $l$ and $\mathrm{u}$ are the known boundary vectors, $l \leq u$. Since the constraints of the problem described in Equation (18) are the upper and lower boundaries of the variables, this can be referred to as a boundary-constrained optimization problem. 
The active-set method is a very effective method by which to solve the boundary constraint problem, and its active set at $\alpha^{*}$ is defined as:

$$
I\left(\alpha^{*}\right)=L\left(\alpha^{*}\right) \cup U\left(\alpha^{*}\right)
$$

In the above equation:

$$
L\left(\alpha^{*}\right)=\left\{i: \alpha_{i}=l_{i}\right\}, U\left(\alpha^{*}\right)=\left\{i: \alpha_{i}=u_{i}\right\}
$$

Clearly, if the active set of Equation (18) is known at the optimal solution, then the problem can be transformed into an equality-constrained optimization problem. However, the optimal solution $\alpha^{*}$ is unknown, thus the active set $I\left(\alpha^{*}\right)$ at $\alpha^{*}$ is unknown, and it is necessary to estimate its active set. This can be determined using a different active set method with a different active set estimation method. In [22-30], different active set methods are discussed in detail, not listed here. The present paper describes the active-set solution method for solving the SHE-PWM equation according to the optimization problem described in Equation (16).

For general SHE-PWM equation problems, as the value of the modulation index $M$ is continuously changing, the trend of the switching angle solution is approximately linear in a range. Therefore, when it is used to solve the optimization problem described in Equation (16), it can find the exact solution at some specific value of $M$, then obtain the solution of the different modulation indexes on both sides of the specific modulation index and determine the solution of the full range through the combination.

Since the numerical iteration method can-not be used to obtain the switching angles with the continuous change mode of the modulation index $M$, the $M$ value is taken at every 0.001 points in the actual solution process. According to the above solution method, it is necessary to first determine the exact solution of $M=0.550,0.650,0.750,0.850,0.950$, then solve the 0.050 range on both sides of the five specific modulation indexes. The process is shown in Figure 11.

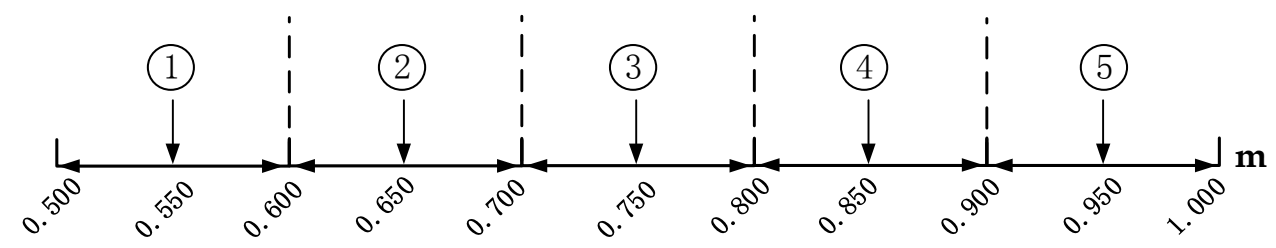

Figure 11. The starting point diagram of the specific modulation index.

When the switching angles of the five specific modulation indexes are calculated, it is difficult to give the initial values of the switching angles, as the switching angle number of Equation (16) is large. This paper uses the second optimization method to improve the initial value problem.

The solving process of the active set of the second optimization method is as follows:

Step 1: The initial value of the switching angle. The initial value given in this paper is arranged by means of permutation and combination. The interval between the two adjacent angles is the same. For example, when the interval is $1^{\circ}$, the switching angles from $\alpha_{1}$ to $\alpha_{7}$ of the first given are $1,2,3,4$, 5,6 , and 7 . For the second time, the initial value of the switching angle $\alpha_{1}$ is incremented by 1 , and the second given initial values are $2,3,4,5,6,7$, and 8 . Under the interval of $1^{\circ}$, the last switch angle reaches $89^{\circ}$, i.e., the interval $1^{\circ}$ precondition, and the final given values are $83,84,85,86,87,88$, and 89 . After the adjacent angle interval is $1^{\circ}$, the interval between the two adjacent angles is increased by 1 , i.e., at intervals of $2^{\circ}$, then the above process is repeated. This is continued so as to increase the interval angle until it reaches $14^{\circ}$, which means that the final given values are $5,19,33,47,61,75$, and 89. For the other three group of switching angles $\alpha_{8}$ to $\alpha_{14}, \alpha_{15}$ to $\alpha_{21}$, and $\alpha_{22}$ to $\alpha_{28}$, the given method of the initial values is same as above. 
Step 2: Use the permutation and combination method to obtain the switching angle initial value table. After determining the initial values of each switching angle group, it is necessary to arrange the initial values of the four switching angle groups to obtain the initial value table of the 28 switching angles in different combinations.

Step 3: The first optimization, in the case of coarse precision, is used to obtain the solution of the switching angles with a specific modulation index. According to the above description, it can be seen that there are many combinations of the switching angle initial values. Therefore, if it requires a large number of iterative algorithms to determine each exact solution, this will greatly increase the computational complexity. It is also possible to enter the local optimum, fail to satisfy the requirements of accuracy, and fall into a "vicious circle" phenomenon. Therefore, when the switching angles are solved under the specific modulation index, this paper uses the second optimization method to solve the problem. Taking the modulation index 0.550 as an example, first, the switching angle initial values are given according to the above initial value given method in the range of $0.550 \pm 0.010$. Then, the optimal results with each modulation index from 0.560 to 0.570 are obtained by using a small number of iterations. In the above solving process, the iteration ends when the specified iteration number is reached. Furthermore, the optimal results obtained in the range above $0.560-0.570$ are filtered, in which the P in the Equation (16) is minimized; this will be the initial value when seeking the exact solution of Equation (16) with the modulation index 0.550.

Step 4: Second optimization: obtain the exact switching angle values for the specific modulation index. The initial values obtained in the third step are taken as the initial values of the modulation index 0.550, and the iterative calculation of Equation (16) is performed using the active set method. Setting the condition of jumping out of iteration is $P \leq 10 \times 10^{-6}$. Finally, the accurate switching angle solution under the modulation index 0.550 can be obtained.

Step 5: Determine the exact solution of the switching angle in the range of \pm 0.050 on both sides of the specific modulation index. The exact switching angle in the case of modulation indexes 0.550 is taken as the initial value, and the solutions of the modulation index in the range of \pm 0.050 on both sides of 0.550 are obtained by using the active-set iterative method. When the modulation index is increasing or decreasing, the initial value of the new modulation index is the exact value of the above time modulation index. Finally, the solution of the switching angle in the range of 0.500 to 0.600 is obtained;

Step 6: Repeat Steps 3 to 5, and replace the specific modulation index followed by $0.650,0.750$, 0.850 , and 0.950 , until the solution of the switching angle in the entire modulation index is obtained.

The above process flow chart is shown in Figure 12. The flow chart only enumerates the solution process when the modulation index is between 0.5 and 0.6 . When solving the switching angle solution of the rest modulation index, it is only necessary to repeat the second optimization process.

In Step 3, first the solution under the coarse precision condition is obtained. This gives the initial value of Step 4 a certain basis, thus greatly reducing the amount of calculation and speeding up the calculation process.

According to the above method, the switching angle solution of Equation (16) with the modulation index $M$ is obtained as shown in Figure 13. 


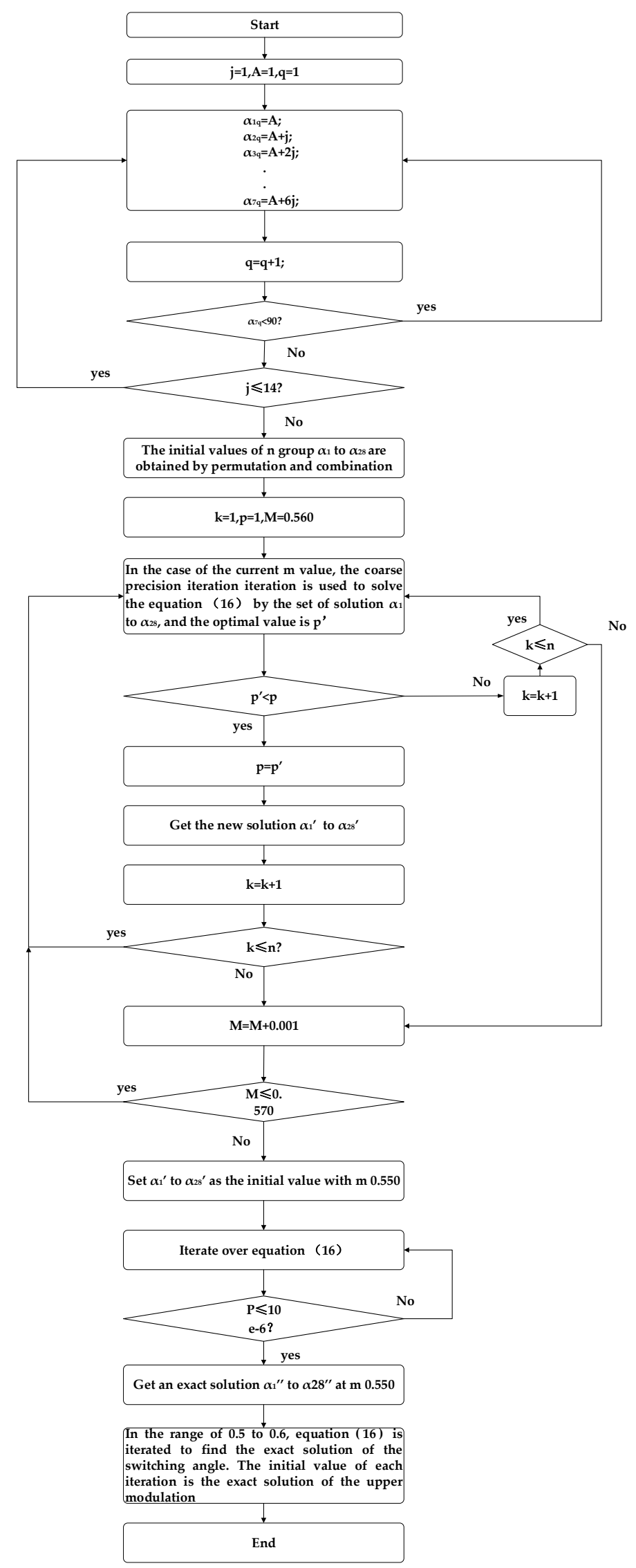

Figure 12. Flow chart of solving process. 

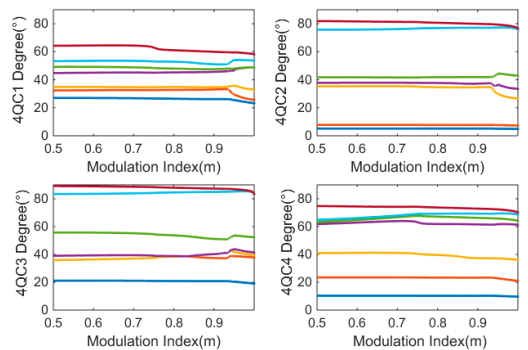

(a)
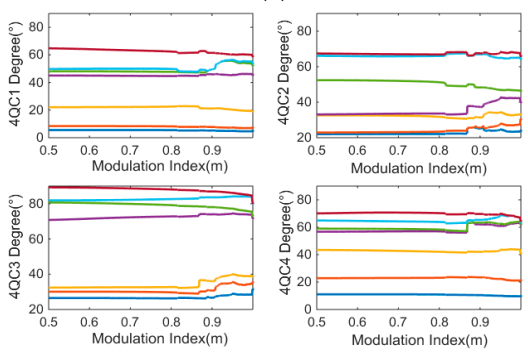

(c)
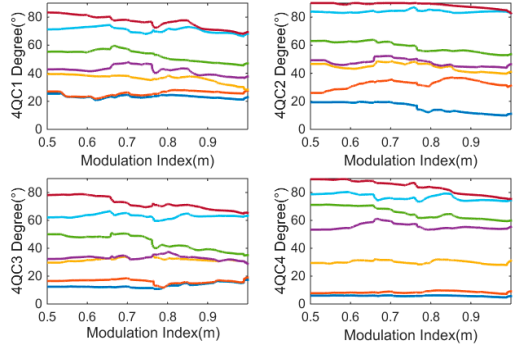

(b)
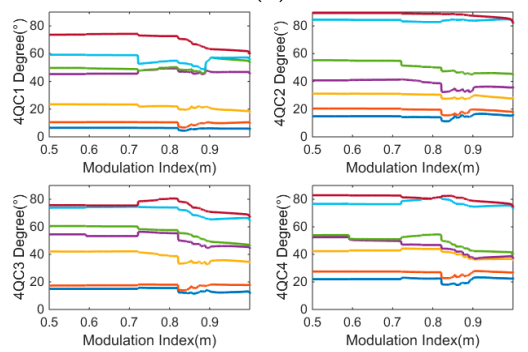

(d)

Figure 13. Switching angle illustration with different elimination harmonics frequency group: (a) 3-35, 37-45; (b) 3-35, 47-55; (c) 3-35, 57-65; and (d) 3-35, 67-75.

\subsection{Specific Harmonic Elimination-Pulse Width Modulation Resonant Suppression Combined with Transient Current Control Strategy}

According to the above calculation results, it can be seen that the linearity of the switch angle solution is poor, and the method of curve fitting will lead to greater errors in some modulation indexes. Therefore, it is more suitable to control quadruple $4 \mathrm{QC}$ by off-line calculation and on-line look-up tables to determine the switching angles. On the basis of Figure 2, the new control block diagram is obtained according to the above SHE-PWM control strategy, as shown in Figure 14.

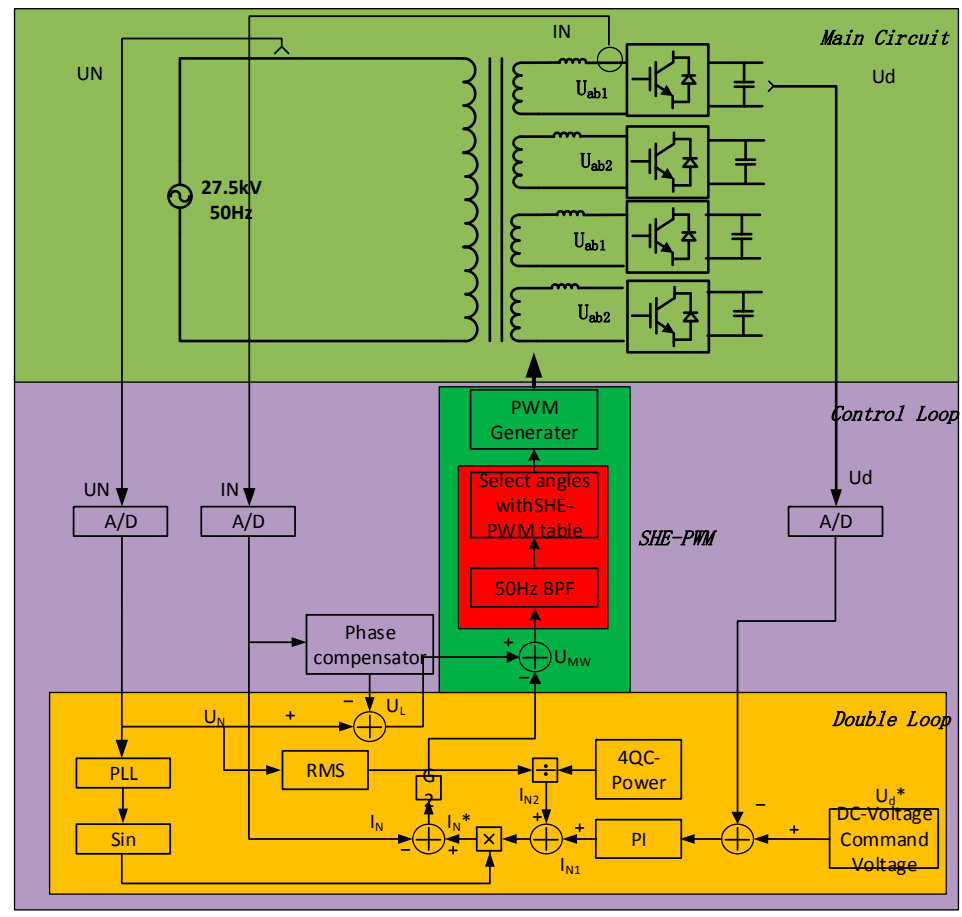

Figure 14. Specific harmonic elimination-pulse width modulation (SHE-PWM) and transient direct current control flow chart. 
As shown in the above figure, the modulated wave signal is first obtained by the double-loop transient direct current control of the voltage outer loop and the inner loop of the current, the amplitude and phase are obtained by a $50 \mathrm{~Hz}$ filter, then, according to the amplitude, the switching angle of the 4QC under this modulation index is obtained by the look-up table method. After this, it can generate pulses to control the quadruple $4 \mathrm{QC}$.

\section{Simulation Results}

Based on the actual parameters of a $4 \mathrm{QC}$ on the train, a simulation model of quadruple $4 \mathrm{QC}$ is built in Matlab to verify the strategy. The simulation parameters are shown in Table 1.

Table 1. Simulation parameters of four quadrant converter.

\begin{tabular}{cc}
\hline Parameter & Value/Unit \\
\hline Transformer primary voltage & $25 \mathrm{kV}$ \\
Transformer secondary voltage & $1658 \mathrm{~V}$ \\
Number of multiplex & 4 \\
DC side support capacitor & $8 \mathrm{pF}$ \\
Switch Frequency & $350 \mathrm{~Hz}$ \\
AC side inductance & $1.195 \mathrm{mH}$ \\
DC side voltage & $2950 \mathrm{~V}$ \\
Power & $1908.4 \mathrm{~kW} \times 4$ \\
\hline
\end{tabular}

\subsection{Simulation of Quadruple $4 Q C$}

Multi 4QC, which adopts carrier phase shifting technology, can obtain better harmonic performance. From Section 2.2, it can be seen that the harmonics of the $4 \mathrm{QC}$ are mainly located near the two-time switching frequency. When multiplex technology is adopted, the effective switching frequency is increased several times. In the quadruple $4 \mathrm{QC}$, the primary current harmonics of the 4QC are concentrated near the eight-times switching frequency, i.e., near the 56th harmonic order $(2800 \mathrm{~Hz})$. The carrier phase shift angles at this time are $0, \pi / 2, \pi / 4$, and $3 \pi / 4$. The Fourier analysis of the primary side current of the transformer is shown in Figure 15.

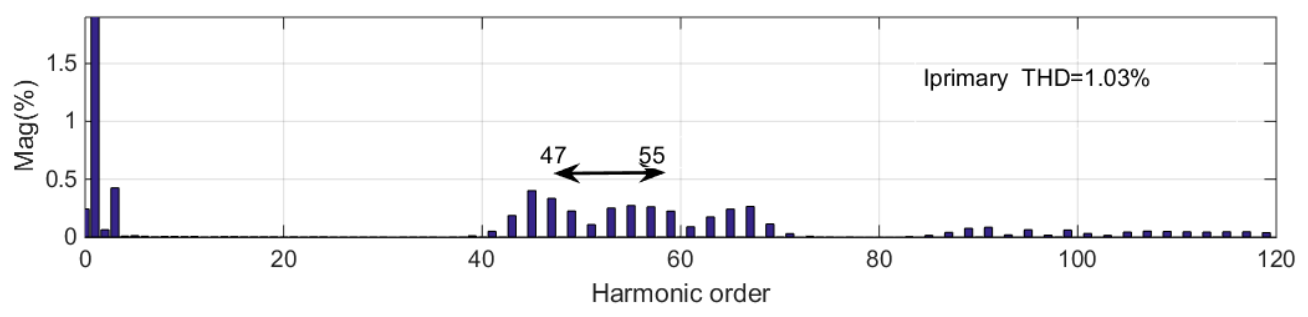

Figure 15. Fourier analysis of transformer primary current.

As can be seen from Figure 15, the harmonic distribution is near the 40th to 70th harmonic order, and only odd harmonics are present. This is consistent with the theoretical analysis of Section 2.2.

\subsection{Verification of Specific Harmonic Elimination-Pulse Width Modulation Resonance Suppression Strategy}

According to the resonance suppression strategy proposed in Sections 3.3 and 3.4, with the above simulation conditions, Band 2.2 is selected as an example. The high-frequency elimination harmonic is selected from the 47th to the 55th harmonic order, and the simulation results are shown in Figures 16 and 17.

Figure 16 shows the transformer primary current and voltage waveforms, and for the sake of comparison, the figure reduces the voltage data by 50 times. As can be seen from Figure 16, the voltage and current phase are the same, and can achieve the unit power factor requirements. 
Figure 17 is a Fourier analysis of the synthetic pulse signal of the quadruple 4QC. As can be seen from Figure 17, in the pulse signal, the third to 35th and 47th to 55th harmonic contents are almost zero, which satisfies the requirements of harmonic elimination. The harmonic signal on both sides of the 47th to 55th harmonic is significantly raised. This is because the elimination harmonic order is 47th to 55th, thus according to the conservation of energy, the harmonics on both sides of the 47 th to 55th harmonics will be raised.

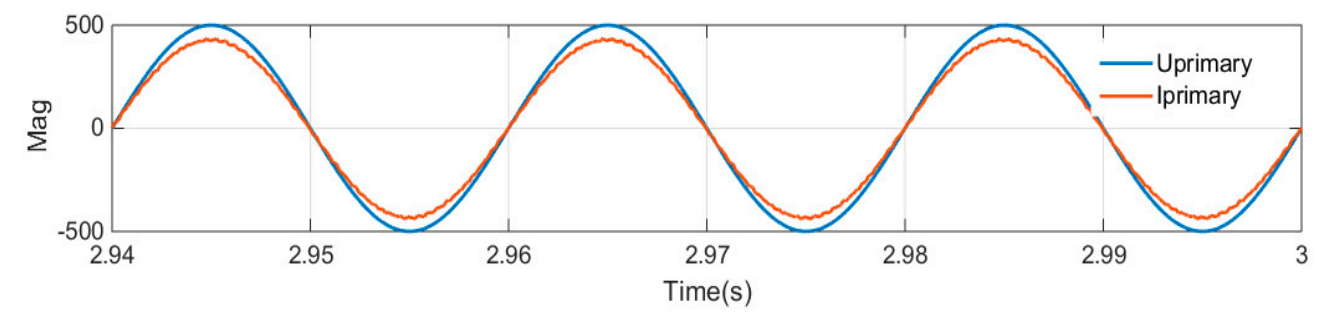

Figure 16. Voltage and current waveform of transformer primary side.
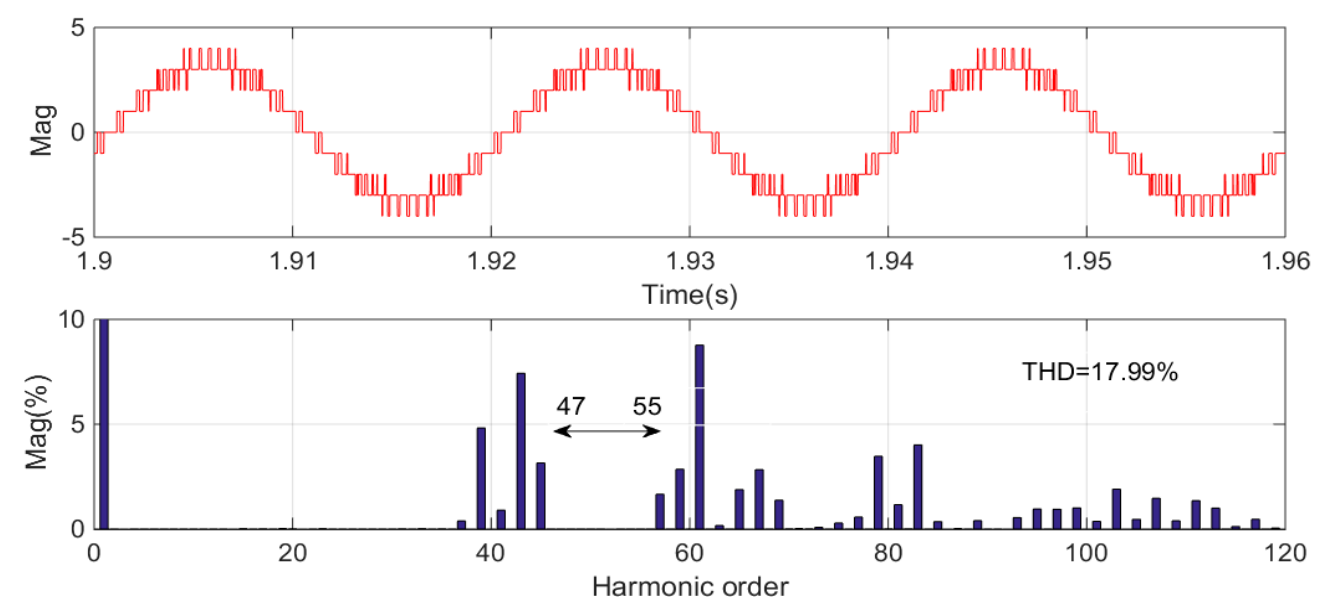

Figure 17. Waveform and Fourier analysis of four-dimensional 4QC synthesis pulse.

Figure 18 is a Fourier analysis of the primary current of the transformer, in which the harmonic content between the 47th and 55th harmonics is almost zero, which achieves the desired aim of eliminating high frequency harmonics. This strategy can effectively avoid the occurrence of resonance in the 47 th to 55 th harmonics range.

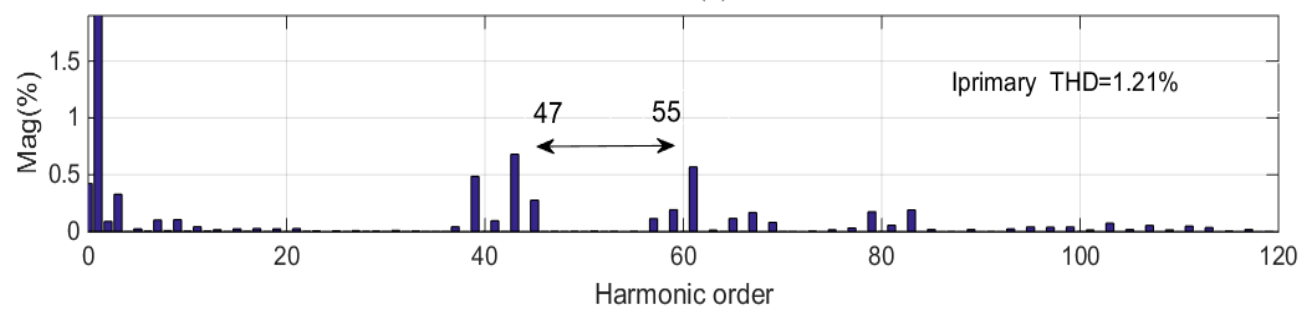

Figure 18. Fourier analysis of transformer primary current.

Upon further observation of Figure 18, it can be seen that the low frequency of the third, fifth, and seventh harmonic elimination effects is relatively poor, and there is a minor difference from Figure 17. The reason for this is that, due to the energy conservation between the DC and AC sides of the 4QC in the simulation, there is a $100 \mathrm{~Hz}$ ripple at the DC side voltage. The DC side pulsation reflects to the 
AC side, resulting in the emergence of low-frequency third, fifth, and seventh harmonics. Solving the second pulsation problem can be achieved by adding a secondary filter on the DC side, or by further improving the Equation (16) to produce a specific harmonic that is opposite to the existing third, fifth, and seventh harmonics.

\subsection{Simulation Verification of Grid-Train Coupling Resonance Suppression}

The five-conductor model is often used to build the traction grid simulation model. However, there are many parameters in this model, which makes it difficult to control the resonant frequency precisely by changing the parameters. Therefore, this paper uses the RLC resonance circuit shown in Figure 19 with a quadruple $4 \mathrm{QC}$ to simulate the grid-train coupling circuit. The resonant frequency of the resonant circuit can be controlled by changing the impedance value of the circuit.

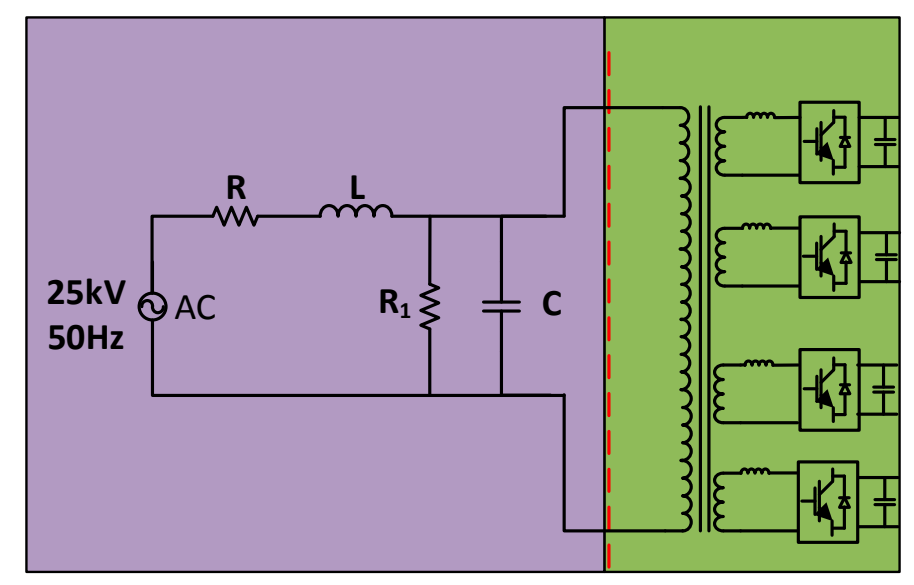

Figure 19. Diagram of grid-train coupling simulation.

The simulation parameters of the $4 \mathrm{QC}$ are the same as those in Section 4.1. At this point, the characteristic harmonic currents of the quadruple 4QC are shown in Figure 15, and its characteristic harmonics are covered from the 40th to 70th harmonic orders. Firstly, the frequency of the resonant circuit in Figure 19 is set to $2500 \mathrm{~Hz}$, i.e., the 50th harmonic order, which is within the range of the abovementioned harmonic band. The impedance analysis of the resonant circuit is shown in Figure 20.

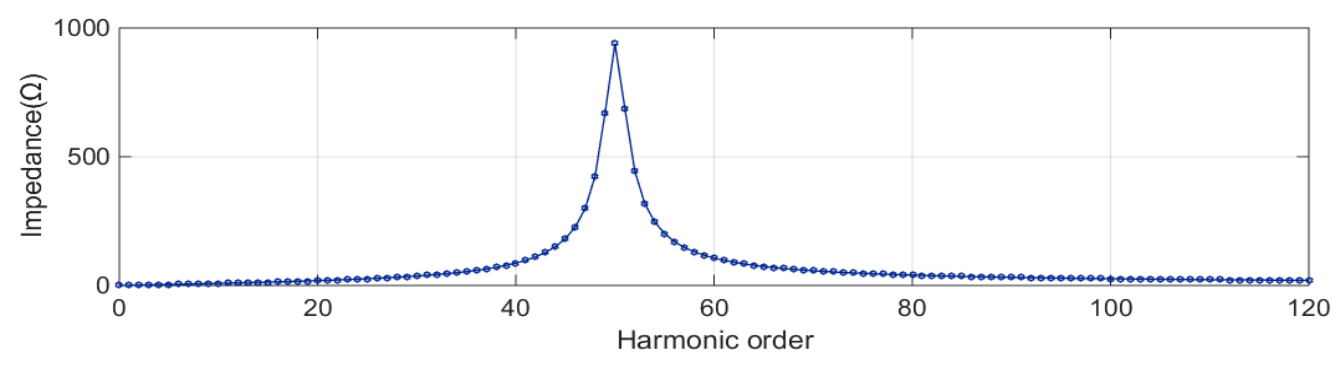

Figure 20. Impedance characteristic diagram of the resonant circuit.

When the quadruple 4QC uses the transient current direct control method, the FFT (Fast Fourier Transform) analysis of the current that is injected into the grid is as shown in Figure 21, while the train port grid voltage waveform and its Fourier analysis are shown in Figure 22. 


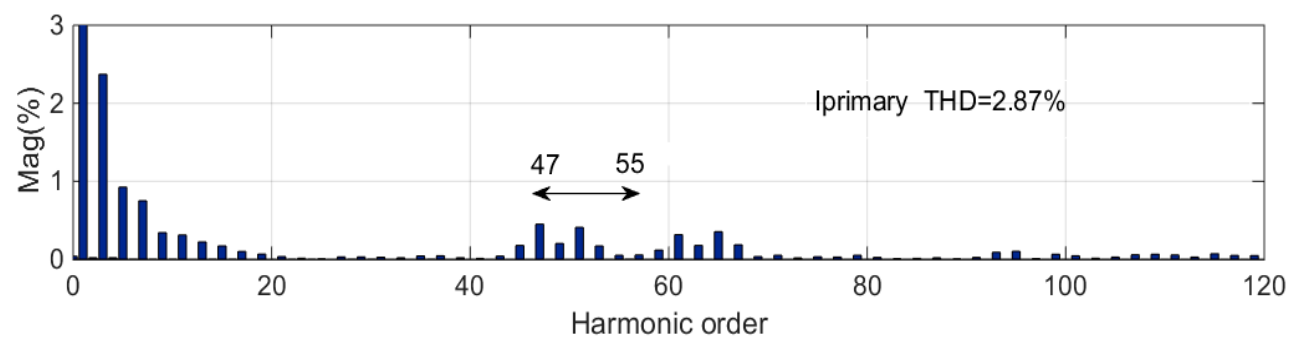

Figure 21. Fourier analysis of transformer primary current.
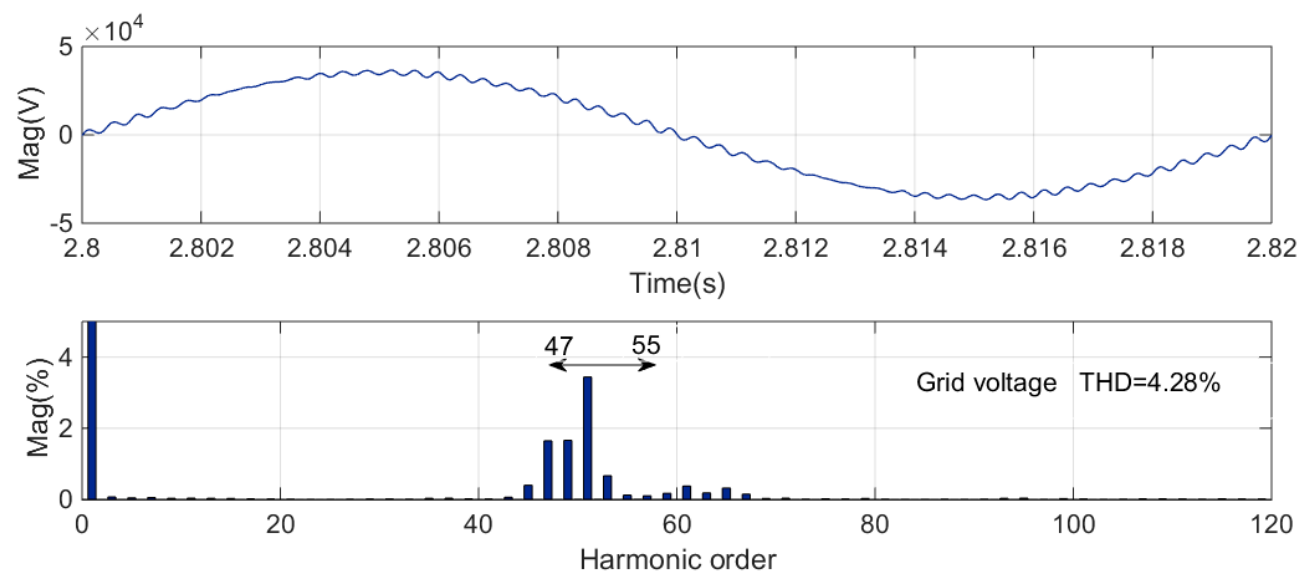

Figure 22. Waveform and FFT analysis of grid voltage.

As can be seen from Figure 21, the current harmonic is concentrated in the range of the 40th to 70th harmonic order, and is similar to Figure 15. The impedance peak frequency is in the range above. The grid voltage waveform produced a certain distortion, the THD value increased significantly, and the grid harmonics are most abundant near the resonant peak of $2500 \mathrm{~Hz}$. Among them, the current contains many low-frequency harmonics because the grid voltage produced a certain distortion, affecting the normal operation of the $4 \mathrm{QC}$.

When the SHE method is used for resonance suppression, select Band 2.2 to eliminate the 47th to 55th harmonics; the FFT analysis of the train current is as shown in Figure 23, and the grid voltage waveform and its FFT analysis are shown in Figure 24.

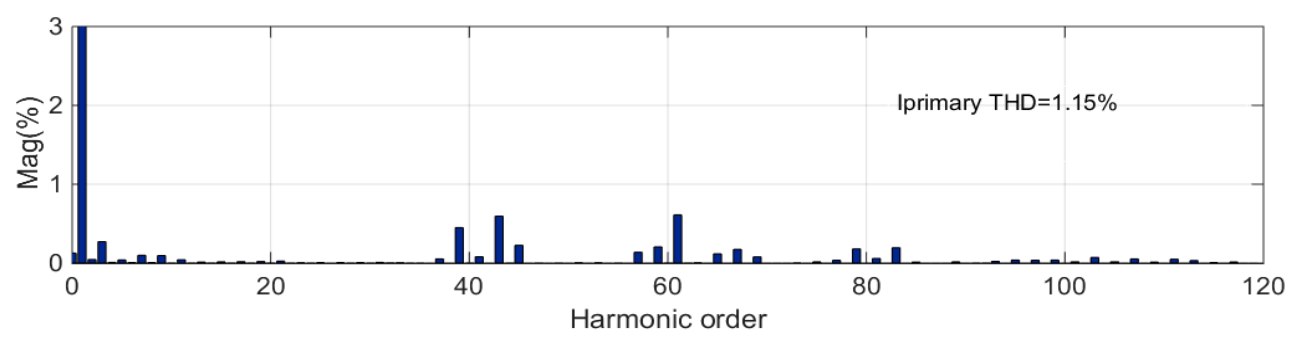

Figure 23. Fourier analysis of transformer primary current with SHE-PWM algorithm. 

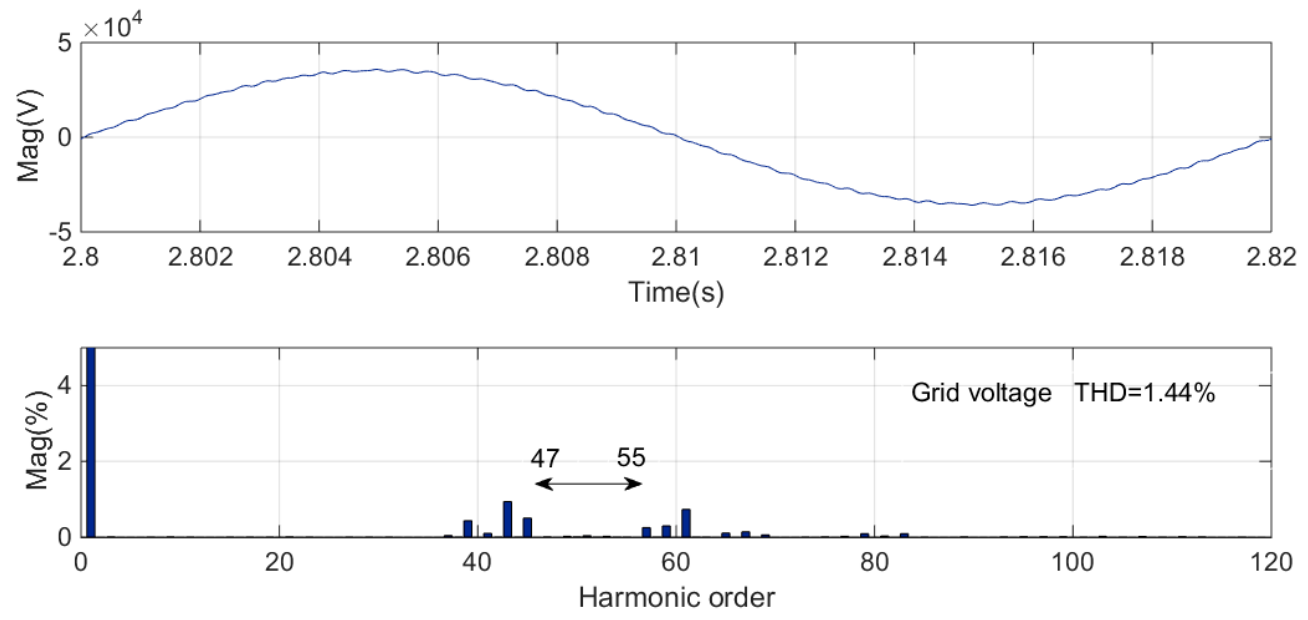

Figure 24. Waveform and FFT analysis of grid voltage with SHE-PWM algorithm.

As can be seen from Figure 23, the 47th to 55th harmonic order elimination effects of the current that is injected into the traction grid are apparent. The distortion of the grid voltage is significantly improved, the voltage THD content is reduced by $66.36 \%$, and the harmonic elimination range is the same as in the theoretical analysis.

As the resonance frequency is set to $3000 \mathrm{~Hz}$, the impedance analysis of the resonant circuit is shown in Figure 25.

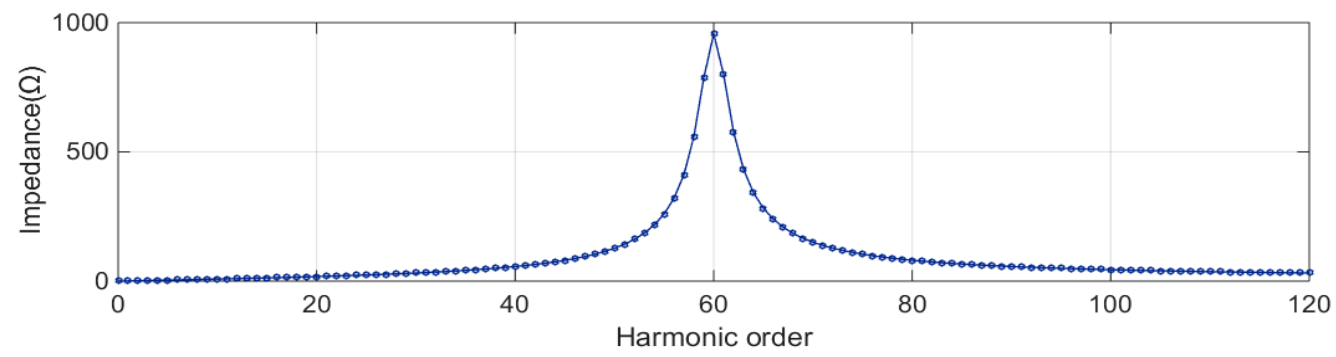

Figure 25. Impedance characteristic diagram of resonant circuit.

The FFT analysis of the current that is injected into the grid is as shown in Figure 26 when the quadruple $4 \mathrm{QC}$ uses the transient current direct control method, while the train port grid voltage waveform and its FFT analysis are shown in Figure 27.

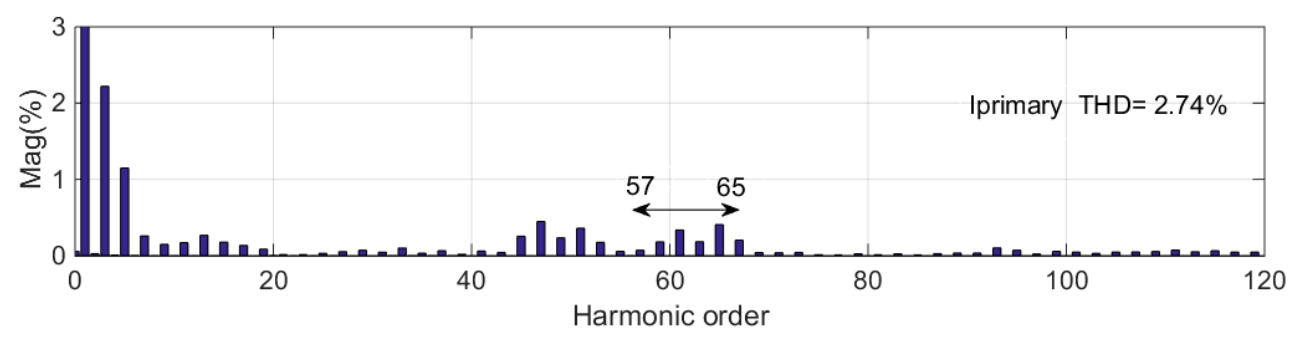

Figure 26. Fourier analysis of transformer primary current. 

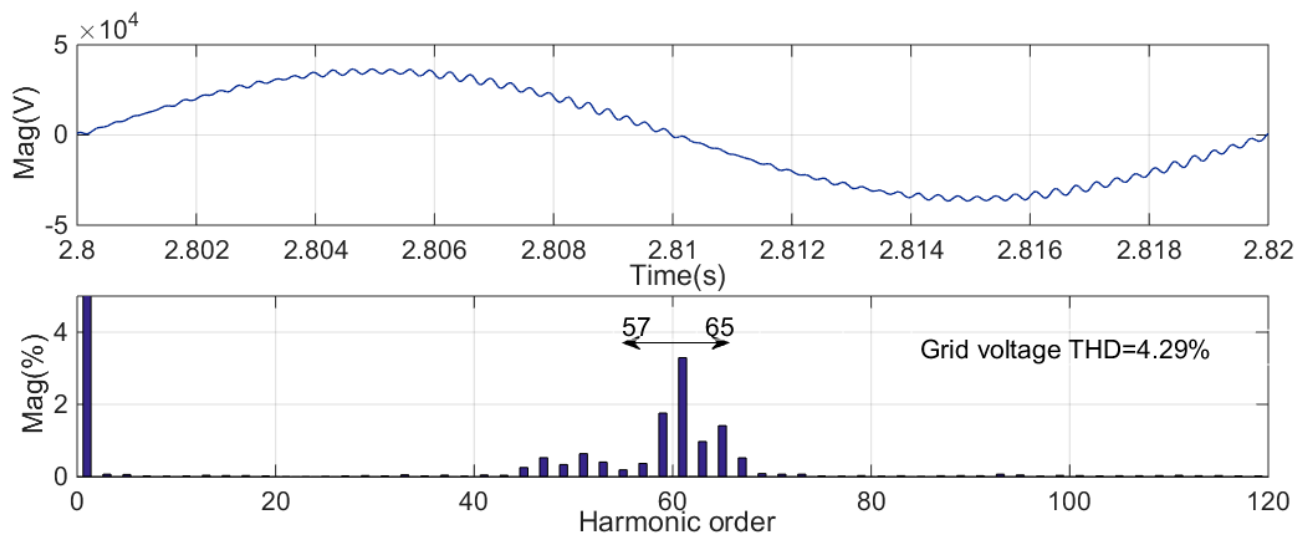

Figure 27. Waveform and FFT analysis of grid voltage.

As can be seen from Figure 27, the harmonic content of the grid voltage obviously increases at the resonant frequency.

Select Band 2.3 to eliminate 57th to 65th harmonics, the FFT analysis of the train current is as shown in Figure 28, and the grid voltage waveform and its FFT analysis are as shown in Figure 29.

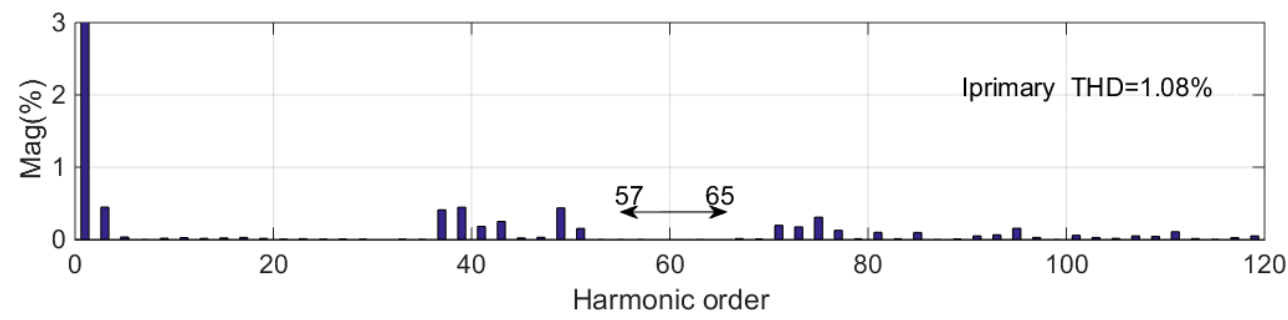

Figure 28. Fourier analysis of transformer primary current with SHE-PWM algorithm.
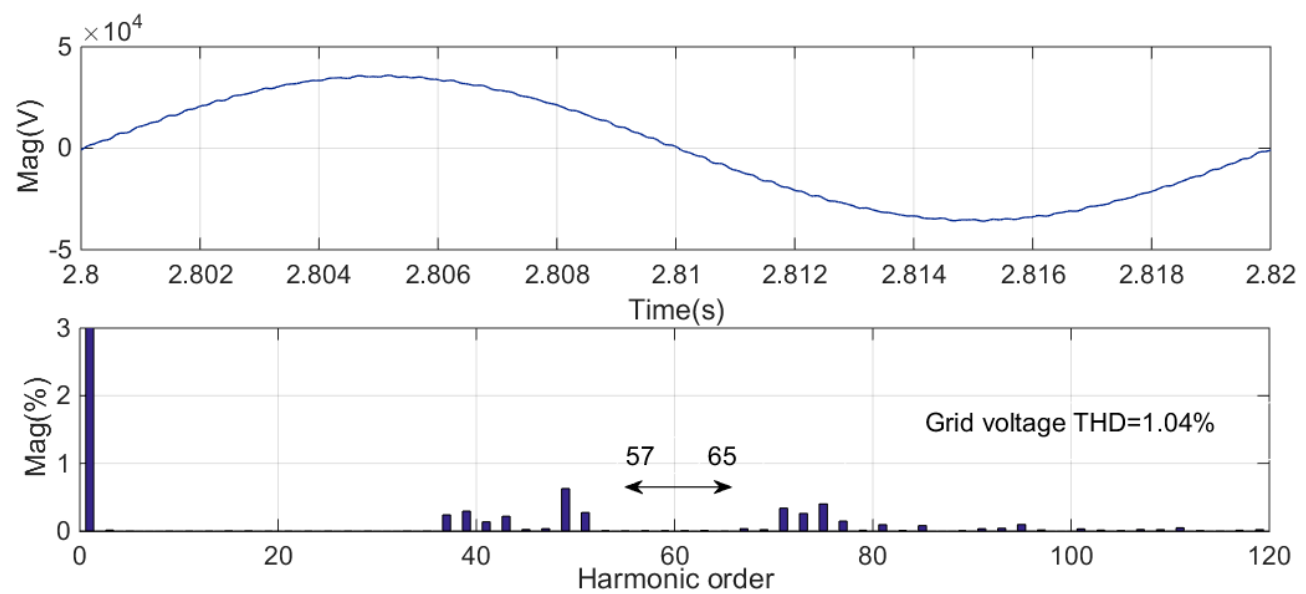

Figure 29. Waveform and FFT analysis of grid voltage with SHE-PWM algorithm.

As can be seen from Figure 28, the 57th to 65th harmonic order elimination effects of the current that is injected into the traction grid are apparent. The distortion of the grid voltage is improved significantly; the voltage THD content is reduced by $75.76 \%$. The resonance suppression effect is remarkable.

The simulation results show that the SHE-PWM strategy is able to suppress the grid-train coupling resonance. 


\section{Conclusions}

In this paper, first, the characteristic harmonic distribution of the $4 \mathrm{QC}$ in the train and the mechanism of the grid-train coupling resonance are analyzed in principle. Then, the SHE-PWM method is combined with the existing transient direct current control, and a special harmonic group elimination strategy is adopted to cover the high-frequency resonant region and improve the current harmonics that are injected into the traction grid. This can allow the current to avoid the resonance frequency of the traction grid, and achieve the purpose of grid-train coupling resonance suppression. At the same time, the active-set secondary optimization method is proposed to solve the transcendental equation composed of a cosine function. The method has the advantages of high precision, fast convergence, and small dependence on initial values. In addition, since the solving process becomes simpler using the above method, SHE-PWM can be further extended to applications where more converters are connected in multiple ways.

However, the DC-side voltage pulsation of the $4 \mathrm{QC}$ results in low-frequency harmonics in the primary current of the transformer, and the method of suppressing the low-frequency harmonics requires further study.

Acknowledgments: This research was supported by the Chinese national key R \& D program 2016YFB1200506-18.

Author Contributions: R.Z. and F.L. conceived and designed the experiments; R.Z. and F.L. performed the experiments; Z.Y., H.C. and Y.L. analyzed the data; H.C. contributed analysis tools; R.Z. wrote the paper.

Conflicts of Interest: The authors declare no conflict of interest.

\section{References}

1. He, Z.; Hu, H.; Zhang, Y.; Gao, S. Harmonic Resonance Assessment to Traction Power-Supply System Considering Train Model in China High-Speed Railway. IEEE Trans. Power Deliv. 2014, 29, 1735-1743. [CrossRef]

2. Chang, G.W.; Lin, H.W.; Chen, S.K. Modeling characteristics of harmonic currents generated by high-speed railway traction drive converters. IEEE Trans Power Deliv. 2004, 19, 766-773. [CrossRef]

3. Cui, H.; Song, W.; Fang, H.; Ge, X.; Feng, X. Resonant harmonic elimination pulse width modulation-based high-frequency resonance suppression of high-speed railways. IET Power Electron. 2015, 8, 735-742. [CrossRef]

4. Song, W.; Jiao, S.; Li, Y.W.; Wang, J.; Huang, J. High-Frequency Harmonic Resonance Suppression in High-Speed Railway Through Single-Phase Traction Converter With LCL Filter. IEEE Trans. Transp. Electr. 2016, 2, 347-356. [CrossRef]

5. Hu, H.; Gao, S.; Shao, Y.; Wang, K.; He, Z.; Chen, L. Harmonic Resonance Evaluation for Hub Traction Substation Consisting of Multiple High-speed Railways. IEEE Trans. Power Deliv. 2017, PP, 1. [CrossRef]

6. Zynovchenko, A.; Xie, J.; Jank, S.; Klier, F. Resonance phenomena and propagation of frequency converter harmonics the catenary of railways with single-phase A.C. In Proceedings of the 2005 European Conference on Power Electronics and Applications, Dresden, Germany, 11-14 September 2005.

7. Liu, M.; Wu, M.; Yang, S.; Chu, Z.; Huang, Z. Traction Harmonic and Transient Overvoltage Suppression Device. Patent CN 101145692 A, 19 March 2008. (In Chinese)

8. Hu, H.; He, Z.; Gao, S. Passive Filter Design for China High-Speed Railway With Considering Harmonic Resonance and Characteristic Harmonics. IEEE Trans. Power Deliv. 2015, 30, 505-514. [CrossRef]

9. Krah, J.O.; Holtz, J. Total compensation of line-side switching harmonics in converter-fed AC locomotives. IEEE Trans. Ind. Appl. 1995, 31, 1264-1273. [CrossRef]

10. Maeda, T.; Watanabe, T.; Mechi, A.; Shiota, T.; Iida, K. A hybrid single-phase power active filter for high order harmonics compensation in converter-fed high speed trains. In Proceedings of the Power Conversion Conference-Nagaoka 1997, Nagaoka, Japan, 6 August 1997; Volume 2, pp. 711-717.

11. Kwon, K.M.; Song, Y.S.; Choi, J. 6MVA single-phase APF for high speed train line in Korea. In Proceedings of the International Conference on Power Engineering and Renewable Energy, Bali, Indonesia, 9-11 December 2014; pp. 31-36. 
12. Bueno, A.; Aller, J.M.; Restrepo, J.A.; Harley, R.; Habetler, T.G. Harmonic and Unbalance Compensation Based on Direct Power Control for Electric Railway Systems. IEEE Trans. Power Electron. 2013, 28, 5823-5831. [CrossRef]

13. Holtz, J.; Krah, J.O. Suppression of time-varying resonances in the power supply line of AC locomotives by inverter control. IEEE Trans. Ind. Electron. 1992, 39, 223-229. [CrossRef]

14. Song, K.; Konstantinou, G.; Wu, M.; Acuna, P.; Aguilera, R.P.; Agelidis, V.G. Windowed SHE-PWM of Interleaved Four-Quadrant Converters for Resonance Suppression in Traction Power Supply Systems. IEEE Trans. Power Electron. 2017, PP, 1. [CrossRef]

15. Patel, H.S.; Hoft, R.G. Generalized Techniques of Harmonic Elimination and Voltage Control in Thyristor Inverters: Part I-Harmonic Elimination. IEEE Trans. Ind. Appl. 1973, IA-9, 310-317. [CrossRef]

16. Routray, A.; Patel, V.; Mahanty, R.; Singh, R.K. A novel GA optimized SHE PWM hybrid cascaded H-bridge multilevel inverter with Capacitor Voltage Averaging for motor drive applications. In Proceedings of the IEEE International Conference on Power Electronics, Drives and Energy Systems, Trivandrum, India, 14-17 December 2016.

17. Aguilera, R.P.; Lezana, P.; Konstantinou, G. Closed-loop SHE-PWM technique for power converters through Model Predictive Control. In Proceedings of the 41st Annual Conference of the IEEE Industrial Electronics Society (IECON 2015), Yokohama, Japan, 9-12 November 2016; pp. 5261-5266.

18. Pulikanti, S.R.; Konstantinou, G.; Agelidis, V.G. Hybrid Seven-Level Cascaded Active Neutral-PointClamped-Based Multilevel Converter Under SHE-PWM. IEEE Trans. Ind. Electron. 2013, 60, 4794-4804. [CrossRef]

19. Dahidah, M.S.A.; Konstantinou, G.; Agelidis, V.G. A Review of Multilevel Selective Harmonic Elimination PWM: Formulations, Solving Algorithms, Implementation and Applications. IEEE Trans. Power Electron. 2015, 30, 4091-4106. [CrossRef]

20. Saponara, S.; Ciarpi, G.; Groza, V.Z. Design and Experimental Measurement of EMI Reduction Techniques for Integrated Switching DC/DC Converters. Can. J. Electr. Comput. Eng. 2017, 40, 116-127.

21. Konstantinou, G.; Agelidis, V.G.; Pou, J. Theoretical Considerations for Single-Phase Interleaved Converters Operated With SHE-PWM. IEEE Trans. Power Electron. 2014, 29, 5124-5128. [CrossRef]

22. Ni, Q.; Yuan, Y. A Subspace Limited Memory Quasi-Newton Algorithm for Large-Scale Nonlinear Bound Constrained Optimization. Math. Comput. Am. Math. Soc. 1997, 66, 1509-1520. [CrossRef]

23. Facchinei, F.; Júdice, J.; Soares, J. An active set Newton algorithm for large-scale nonlinear programs with box constraints. SIAM J. Optim. 1995, 8, 158-186. [CrossRef]

24. Facchinei, F.; Lucidi, S.; Palagi, L. A Truncated Newton Algorithm for Large Scale Box Constrained Optimization. SIAM J. Optim. 2002, 12, 1100-1125. [CrossRef]

25. Sun, L.; He, G.; Wang, Y.; Fang, L. An active set quasi-Newton method with projected search for bound constrained minimization. Comput. Math. Appl. 2009, 58, 161-170. [CrossRef]

26. Hintermüller, M.; Ito, K.; Kunisch, K. The Primal-Dual Active Set Strategy as a Semismooth Newton Method. SIAM J. Optim. 2002, 13, 865-888. [CrossRef]

27. Krongold, B.S.; Jones, D.L. An active-set approach for OFDM PAR reduction via tone reservation. IEEE Trans. Signal Process. 2004, 52, 495-509. [CrossRef]

28. Birgin, E.G.; Martínez, J.M. Large-Scale Active-Set Box-Constrained Optimization Method with Spectral Projected Gradients. Comput. Optim. Appl. 2002, 23, 101-125. [CrossRef]

29. Ito, K.; Kunisch, K. The Primal-Dual Active Set Method for Nonlinear Optimal Control Problems with Bilateral Constraints. SIAM J. Control Optim. 2004, 43, 357-376. [CrossRef]

30. Virtanen, T.; Gemmeke, J.F.; Raj, B. Active-Set Newton Algorithm for Overcomplete Non-Negative Representations of Audio. IEEE Trans. Audio Speech Lang. Process. 2013, 21, 2277-2289. [CrossRef]

(C) 2017 by the authors. Licensee MDPI, Basel, Switzerland. This article is an open access article distributed under the terms and conditions of the Creative Commons Attribution (CC BY) license (http://creativecommons.org/licenses/by/4.0/). 